\title{
Higher-twist fermionic operators and DIS structure functions from the AdS/CFT duality
}

\author{
David Jorrin* and Martin Schvellinger@ $^{\dagger}$ \\ Instituto de Física La Plata-UNLP-CONICET, Boulevard 113 e 63 y 64, \\ (1900) La Plata, Buenos Aires, Argentina, \\ and Departamento de Física, Facultad de Ciencias Exactas, Universidad Nacional de La Plata, \\ Calle 49 y 115, C.C. 67, (1900) La Plata, Buenos Aires, Argentina
}

(Received 19 October 2020; accepted 9 April 2021; published 11 May 2021)

\begin{abstract}
The role of local higher-twist $(\tau>3)$ spin-1/2 fermionic operators of the strongly coupled $\mathcal{N}=4$ supersymmetric Yang-Mills theory on the symmetric and antisymmetric deep inelastic scattering structure functions is investigated. The calculations are carried out in terms of the duality between $\mathcal{N}=4$ SYM theory and type IIB supergravity on $\mathrm{AdS}_{5} \times S^{5}$. Particularly, we explicitly obtain the structure functions for single-trace spin- $1 / 2$ fermionic operators in the $\mathbf{2 0}^{*}$ and $\mathbf{6 0}^{*}$ irreducible representations of $S U(4)_{R}$, corresponding to twists 4 and 5, respectively. We also calculate the contributions of other single-trace spin-1/2 fermionic operators in the 4, 20 and $\mathbf{6 0}$ irreducible representations of $S U(4)_{R}$. New important effects are found in comparison with the minimal twist $(\tau=3)$ case, and they are studied thoroughly.
\end{abstract}

DOI: 10.1103/PhysRevD.103.106012

\section{INTRODUCTION}

Deep inelastic scattering (DIS) cross sections of charged leptons by hadrons are expressed as the contraction of a leptonic tensor with a hadronic one. The leptonic tensor is easily obtained from QED. The problem lies within the calculation of the hadronic tensor, which is given in terms of the two-point function of electromagnetic currents within the hadron, where strong coupling effects become important. In the operator product expansion (OPE) of two electromagnetic currents inside a hadron there are several kinds of contributions from different SYM theory operators, which in certain parametric domains can be relevant depending on the virtual-photon momentum $q$, the coupling $g_{\mathrm{SYM}}$, the Bjorken parameter $x$, and the number of color degrees of freedom $N$. Certain properties of the hadronic tensor as well as relations among different structure functions, such as the Callan-Gross relation and generalizations of it, are valid for different gauge field theories, at least within the same parametric regimes of $q$, $g_{\mathrm{SYM}}, x$, and $N$. In particular, this behavior has been found in the framework of the gauge/string theory duality [1-3] in diverse situations for the strongly coupled regime of

\footnotetext{
jorrin@ fisica.unlp.edu.ar martin@fisica.unlp.edu.ar
}

Published by the American Physical Society under the terms of the Creative Commons Attribution 4.0 International license. Further distribution of this work must maintain attribution to the author(s) and the published article's title, journal citation, and DOI. Funded by SCOAP ${ }^{3}$. different gauge theories, starting from the pioneering work by Polchinski and Strassler [4]. Structure functions of spin- $1 / 2$ hadrons have been investigated in this context in [4-10]. These techniques have been also applied to the study of the structure functions of scalar and vector mesons from Dp-brane systems with flavor branes preserving some supersymmetries as in the D3D7-brane model [11], or breaking supersymmetry completely as in the SakaiSugimoto model [12] and in the D4D6 anti-D6-brane model [13], which have been considered in [14-20]. Also, $1 / N$ corrections have been investigated in this context [21-23]. In addition, very important holographic Pomeron techniques have been developed and applied to different models derived from both type IIA and type IIB superstring theories [24-31]. Another interesting related aspect is the DIS off a strongly coupled $\mathcal{N}=4$ SYM plasma [32], as well as its corrections within the strong coupling expansion which have been obtained in [33] from $\alpha^{13}$ string theory corrections to the type IIB supergravity action [34].

For the electromagnetic DIS let us consider an incident polarized spin-1/2 hadron, with four-momentum $P^{\mu}$, mass $M$, and a spin vector $S^{\mu}$. The corresponding hadronic tensor can be written as

$$
W_{\mu \nu}=W_{\mu \nu}^{(S)}(q, P)+i W_{\mu \nu}^{(A)}(q, P, s),
$$

which is expressed in terms of the Bjorken variable defined as $x=-q^{2} /(2 P \cdot q)$. The DIS limit corresponds to $q \rightarrow \infty$, while $x$ is kept fixed. The hadronic tensor can be written in terms of the structure functions as follows $[35,36]$ : 


$$
\begin{aligned}
W_{\mu \nu}^{(S)}= & \left(\eta_{\mu \nu}-\frac{q_{\mu} q_{\nu}}{q^{2}}\right)\left[F_{1}\left(x, q^{2}\right)+\frac{M S \cdot q}{2 P \cdot q} g_{5}\left(x, q^{2}\right)\right] \\
& -\frac{1}{P \cdot q}\left(P_{\mu}-\frac{P \cdot q}{q^{2}} q_{\mu}\right)\left(P_{\nu}-\frac{P \cdot q}{q^{2}} q_{\nu}\right)\left[F_{2}\left(x, q^{2}\right)+\frac{M S \cdot q}{P \cdot q} g_{4}\left(x, q^{2}\right)\right] \\
& -\frac{M}{2 P \cdot q}\left[\left(P_{\mu}-\frac{P \cdot q}{q^{2}} q_{\mu}\right)\left(S_{\nu}-\frac{S \cdot q}{P \cdot q} P_{\nu}\right)+\left(P_{\nu}-\frac{P \cdot q}{q^{2}} q_{\nu}\right)\left(S_{\mu}-\frac{S \cdot q}{P \cdot q} P_{\mu}\right)\right] g_{3}\left(x, q^{2}\right), \\
W_{\mu \nu}^{(A)}= & -\frac{M \epsilon_{\mu \nu \rho \sigma} q^{\rho}}{P \cdot q}\left(S^{\sigma} g_{1}\left(x, q^{2}\right)+\left[S^{\sigma}-\frac{S \cdot q}{P \cdot q} P^{\sigma}\right] g_{2}\left(x, q^{2}\right)\right)-\frac{\epsilon_{\mu \nu \rho \sigma} q^{\rho} P^{\sigma}}{2 P \cdot q} F_{3}\left(x, q^{2}\right),
\end{aligned}
$$

where we have separated the hadronic tensor into its symmetric and antisymmetric parts, and also we have used the metric defined as $\eta_{\mu \nu}=\operatorname{diag}(-1,1,1,1)$. In addition, one can define another tensor $T^{\mu \nu}$, related to the forward Compton scattering, as the expectation value of the timeordered product of two electromagnetic currents inside the hadron,

$T^{\mu \nu} \equiv i \int d^{4} \xi e^{i q \cdot \xi}\left\langle P, \mathcal{Q}, S\left|\hat{T}\left\{J^{\mu}(\xi) J^{\nu}(0)\right\}\right| P, \mathcal{Q}, S\right\rangle$

Its imaginary part can be expressed as a sum over intermediate states that we call $\mathcal{X}$,

$$
\begin{aligned}
\operatorname{Im}\left(T^{\mu \nu}\right)= & 2 \pi^{2} \sum_{\mathcal{X}} \delta\left(M_{\mathcal{X}}^{2}+(P+q)^{2}\right) \\
& \times\left\langle P, \mathcal{Q}, S\left|J^{\nu}(0)\right| P_{\mathcal{X}}, \mathcal{Q}, S\right\rangle \\
& \times\left\langle P_{\mathcal{X}}, \mathcal{Q}, S\left|J^{\mu}(0)\right| P, \mathcal{Q}, S\right\rangle .
\end{aligned}
$$

In terms of the optical theorem we have

$$
W_{\mu \nu}^{(S)}=2 \pi \operatorname{Im}\left(T_{\mu \nu}^{(S)}\right), \quad W_{\mu \nu}^{(A)}=2 \pi \operatorname{Im}\left(T_{\mu \nu}^{(A)}\right) .
$$

In the case of the planar limit of the strongly coupled $\mathcal{N}=4$ SYM theory with gauge group $S U(N)$, when $1 \ll \lambda_{\text {SYM }} \ll N$ one can explicitly calculate the hadronic tensor from its string theory dual description, given in terms of type IIB superstring theory on $\mathrm{AdS}_{5} \times S^{5}$ in the $\alpha^{\prime} \rightarrow 0$ limit, i.e., type IIB supergravity, including an IR cutoff $\Lambda$ in order to account for color confinement [4]. In particular, when the Bjorken variable is in the $\lambda_{\mathrm{SYM}}^{-1 / 2} \ll x<1$ regime (where the 't Hooft coupling is $\lambda_{\mathrm{SYM}} \equiv g_{\mathrm{SYM}}^{2} N$ ), only type IIB supergravity fields are relevant for the holographic dual calculation of properties related to the DIS. In that parametric region the OPE of the two electromagnetic currents inside the hadron is dominated by double-trace operators obtained as the product of two protected singletrace operators. There is a factorization in terms of $\left(\Lambda^{2} / q^{2}\right)^{\tau-1}$. The twist is defined as $\tau=\Delta-s$, for an operator with scaling dimension $\Delta$ and spin $s$. In a previous paper [10] we have considered single-trace spin-1/2 fermionic operators with $\tau=3$ which belong to the $\mathbf{4}^{*}$ irreducible representation of $S U(4)_{R}$. For that purpose first we have derived the corresponding terms in the effective five-dimensional supergravity action containing the coupling of two dilatino modes with a massless vector field. We have done it from the dimensional reduction of type IIB supergravity on $S^{5}$. Those terms in the five-dimensional action, which we briefly discuss in Sec. III of the present work, are the minimal coupling and two Pauli terms, one of which connects the same incoming and intermediate states (in the forward Compton scattering related to the DIS process via the optical theorem) and a second one which allows for certain different intermediate states that we study in detail. In [10] we have shown that for $\tau=3$ spin-1/2 fermionic operators the effects due to Pauli terms account for about $90 \%$ of each structure function, thus they play a very important role in the DIS process of $S U(N) \mathcal{N}=4$ SYM theory at strong coupling in the planar limit.

In the present work we investigate the contributions given by local single-trace higher-twist $(\tau>3)$ spin-1/2 fermionic operators of the strongly coupled $S U(N) \mathcal{N}=4$ SYM theory on both the symmetric and the antisymmetric structure functions of a polarized spin-1/2 hadron. We consider the large $N$ limit. We work within the supergravity parametric domain, thus we consider the spontaneous compactification of type IIB supergravity on $S^{5}$. We focus on the structure functions related to twist $\tau=k+3$ spin-1/2 fermionic operators of the type $\mathcal{O}_{k}^{I_{k},(6)}$ defined in Sec. II. Our special interest is in the cases of twists 4 and 5 , corresponding to $k=1$ and 2 , respectively. In the calculation we also discuss the effect of the $\mathcal{O}_{k}^{I_{k},(13)}$ singletrace spin-1/2 fermionic operators which, by virtue of the selection rules we found, also appear as possible final states in the DIS process we consider. It is interesting to emphasize that for single-trace higher-twist spin-1/2 operators there are important new effects that we investigate in this work. One of such effects comes from the fact that as $k$ increases the dimension of the irreducible representation of $S U(4)_{R}$ increases substantially leading to a large number of Kaluza-Klein dilatino modes contributing from the 
supergravity side. For instance, for $\tau=4$ there are 20 Kaluza-Klein modes related to the type IIB supergravity dilatino modes on $\mathrm{AdS}_{5} \times S^{5}$ which contribute to the calculation. Things become even much more complicated for $\tau=5$ where there are 60 spinors contributing to the calculation of the structure functions. In Sec. II we discuss the relation between SYM operators and Kaluza-Klein dilatino modes in each case. On the other hand, there are new additional terms providing relevant contributions coming from the fact that for $\tau>3$ the selection rule $\lambda_{k} \rightarrow \lambda_{k-1}$ with $k>0$ now plays a significant role. These contributions are not subleading in comparison with the contributions that appear for $\tau=3$ spin- $1 / 2$ fermionic operators. Therefore, it is worth investigating the effect of all these new contributions altogether on the hadronic tensor of spin- $1 / 2$ fermions. We will carry out a detailed calculation of the referred effects. This is very interesting because it allows us to understand better how the supergravity dual description accounts for the way the momentum fragmentation and evolution occur in the planar limit of the strongly coupled quantum field theory within the $\lambda_{\text {SYM }}^{-1 / 2} \ll x<1$ range for spin- $1 / 2$ fermionic operators of $\mathcal{N}=4$ SYM theory.

The structure of this work is as follows. In Sec. II we describe the relation between single-trace spin- $1 / 2$ fermionic SYM theory operators and the Kaluza-Klein field modes obtained by considering the dimensional reduction of type IIB supergravity on $S^{5}$. In Sec. III we develop the dual type IIB supergravity calculation of the structure functions for the mentioned operators for twist 4 in Sec. III A and for twist 5 in Sec. III B. In Sec. IV we analyze our results and present the conclusions. There are in addition several Appendixes containing certain important details of the calculations.

\section{SPIN-1/2 FERMIONIC OPERATORS OF $\mathcal{N}=4$ SYM AND TYPE IIB SUPERGRAVITY FIELDS}

The $\mathcal{N}=4$ SYM gauge supermultiplet contains four left Weyl fermions which we label as $\lambda_{\mathcal{N}=4}$ (we use this notation to distinguish it from $\hat{\lambda}$ which represents the tendimensional dilatino field of type IIB supergravity). There are also $X_{j}$ real scalars with $j=1, \ldots, 6$, and $F_{+}$labels the self-dual two-form field strength associated with the $S U(N)$ gauge field. All these fields transform in the adjoint representation of the gauge group $S U(N)$.

We focus on the structure functions corresponding to local twist $\tau=k+3$ spin-1/2 fermionic operators of the form $\mathcal{O}_{k}^{I_{k},(6)}(x)=C_{i_{1} \cdots i_{k}}^{I_{k},(6)} \operatorname{Tr}\left(F_{+} \lambda_{\mathcal{N}=4} X_{i_{1}} \ldots X_{i_{k}}\right)(x)$ where $i_{j}$ are indices corresponding to the six real scalars of the $\mathcal{N}=4$ SYM gauge supermultiplet. In addition, the integer $I_{k}$ runs from 1 to the dimension of the irreducible representation of $S U(4)_{R}$. These operators transform in the $[1, k, 0]$ irreducible representation of the $R$-symmetry group of the $\mathcal{N}=4 \mathrm{SYM}$ theory, being $k \geq 0$ (see for instance [37,38] and references therein). The case for $k=0$ has been investigated in detail in [10]. In that situation there are just four operators of the form $\mathcal{O}_{k=0}^{I_{0},(6)}(x)=C^{I_{0},(6)} \operatorname{Tr}\left(F_{+} \lambda_{\mathcal{N}=4}\right)(x)$, corresponding to $\tau=3$, which are in the $4^{*}$ irreducible representation of $S U(4)_{R}$. As the number of scalar fields becomes larger the complexity of the calculation increases dramatically since the dimension of the corresponding irreducible representation grows with $k=0,1,2,3,4, \ldots$ as $4,20,60,140,280, \ldots$. This means that in terms of the AdS/CFT duality one has to deal with an increasing number of operators on the gauge theory side, and also with the same number of Kaluza-Klein dilatino modes on the type IIB supergravity side. For this reason, and in order to show explicitly the new effects we find for higher-twist operators we only carry out the explicit calculations of the hadronic tensor in the cases of twist-4 and twist-5 spin-1/2 fermionic operators. For higher-twist operators $\mathcal{O}_{k}^{I_{k},(6)}(x)$ the same method can be applied.

In order to calculate the dimension of an irreducible representation of $S U(4)_{R}$ it is useful to consider in general the irreducible representations of the $s u(n)$ algebra of the $S U(n)$ Lie group. Recall that a simple Lie algebra has a Cartan subalgebra of rank $r$ and an associated root space spanned in a basis given by the corresponding simple roots, $\alpha_{i}$, with $i=1, \ldots, r$. There is also a reciprocal basis of vectors $\beta^{j}, j=1, \ldots, r$. An irreducible representation of the Lie algebra can be described in terms of its highest weight vector $V=\sum_{j=1}^{r} m_{j} \beta^{j}$, where $m_{j}$ are the Dynkin integers labeling the different irreducible representations $\left[m_{1}, m_{2}, \ldots, m_{r}\right]$ of $s u(n)$. The dimension of $\left[m_{1}, m_{2}, \ldots, m_{r}\right]$ can be calculated very easily by associating a Young diagram with that representation as follows. One must construct a Young diagram with $m_{j}$ columns of length $j$ (the length is given by the number of single boxes in that column). The relation between $n$ and $r$ is $n=r+1$, thus for the Lie group $S U(4)_{R}$ the rank of its Cartan subalgebra is $r=3$, i.e., there are only three simple roots, therefore the irreducible representations of $S U(4)_{R}$ can be labeled by three Dynkin integers $\left[r_{1}, r_{2}, r_{3}\right] .{ }^{1}$ In particular, for the single-trace spin- $1 / 2$ fermionic operators $\mathcal{O}_{k}^{I_{k},(6)}(x)$ the irreducible representations of $S U(4)_{R}$ are $[1, k, 0]$ with $k \geq 0$. Also, these operators transform in the $(1 / 2,0)$ representation of the algebra of $S U(2) \times S U(2)$ which is isomorphic to the complexified algebra of the Lorentz group $S O(3,1)$, while their conformal dimensions are $\Delta=k+\frac{7}{2}$.

On the other hand, let us recall that the type IIB supergravity spontaneous compactification on $\mathrm{AdS}_{5} \times S^{5}$ for the ten-dimensional dilatino leads to two towers of five-dimensional Kaluza-Klein dilatino modes, $\lambda_{k}^{ \pm}$, whose

\footnotetext{
${ }^{1}$ Notice that we have now switched to the standard notation by calling $r_{j}$ to the Dynkin labels of the irreducible representation of the $S U(4)_{R}$ Lie group, i.e., $\left[m_{1}, m_{2}, m_{3}\right] \equiv\left[r_{1}, r_{2}, r_{3}\right]$.
} 
five-dimensional masses are $m_{k}^{+}=k+\frac{7}{2}$ and $m_{k}^{-}=-k-\frac{3}{2}$, respectively [39-41]. These spinor spherical harmonics on $S^{5}$ are labeled by a set of five positive integers $\left(l_{1}, l_{2}, l_{3}, l_{4}, l_{5}\right)$, which fulfill the relations $l_{5} \geq l_{4} \geq l_{3} \geq$ $l_{2} \geq l_{1} \geq 0$. Also, notice that there is the identification $l \equiv l_{5} \equiv k$. Recall that the second Dynkin integer $r_{2}$ is now $k$. The degeneracies of the above five-dimensional dilatino modes are given by

$$
d_{\text {spinor }}(5, k)=4\left[\left(\begin{array}{c}
5+k \\
k
\end{array}\right)-\left(\begin{array}{c}
5+k-1 \\
k-1
\end{array}\right)\right],
$$

always for $k \geq 0$.

Now, let us work out some relevant examples for us. Consider first the case $k=0$. The dimension of the $[1,0,0]$ representation is given by the ratio between the values of the following Young tableaux: 4 and 1, whose values are 4 and 1, respectively. This corresponds to the degeneracy of the mass $m_{0}^{-}=-3 / 2$ given by Eq. (2.1), i.e., there are four dilatino modes corresponding to the $(0,0,0,0,0)_{a}$ spinor spherical harmonics of $S^{5}$. The subindex $a=1, \ldots, 4$ labels each of these spinor spherical harmonics.

For $k=1$ we have $\mathcal{O}_{k=1}^{I_{1},(6)}(x)=C_{i_{1}}^{I_{1},(6)} \operatorname{Tr}\left(F_{+} \lambda_{\mathcal{N}=4} X_{i_{1}}\right)(x)$, corresponding to $\tau=4$, and these operators are in the $\mathbf{2 0}^{*}$ irreducible representation of $S U(4)_{R}$, which is labeled as $[1,1,0]$. Its dimension is given by the ratio between the values of the following Young tableaux:

$$
\begin{array}{|l|l|l|}
\hline 4 & 5 \\
\hline 3 &
\end{array} \text { and } \begin{array}{|l|l|}
\hline 3 & 1 \\
\hline 1 & \\
\hline
\end{array}
$$

whose values are $4 \cdot 5 \cdot 3$ and $3 \cdot 1 \cdot 1$, respectively, thus obtaining 20 as the dimension of this representation. This number is the same as the number of the mass degeneracy of the corresponding five-dimensional dilatino modes given by $(1,1,1,1,1)_{a},(1,1,1,1,0)_{a}$, $(1,1,1,0,0)_{a},(1,1,0,0,0)_{a}$ and $(1,0,0,0,0)_{a}$, each of which has four spinors associated.

When $k=2$ the operators are $\mathcal{O}_{k=2}^{I_{2},(6)}(x)=$ $C_{i_{1} i_{2}}^{I_{2},(6)} \operatorname{Tr}\left(F_{+} \lambda_{\mathcal{N}=4} X_{i_{1}} X_{i_{2}}\right)(x)$, which correspond to $\tau=5$, being operators in the $\mathbf{6 0}^{*}$ irreducible representation of $S U(4)_{R}$. In this case this is the $[1,2,0]$ representation. Now, the dimension is given by the ratio between the values of the following Young tableaux:

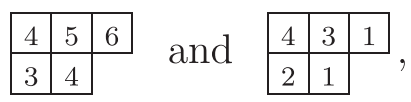

whose values are $4 \cdot 5 \cdot 6 \cdot 3 \cdot 4$ and $4 \cdot 3 \cdot 1 \cdot 2 \cdot 1$, respectively, which gives 60 . This number corresponds to the mass degeneracy of the corresponding five-dimensional dilatino modes:
$(2,2,2,2,2)_{a}$,
$(2,2,2,2,1)_{a}$,
$(2,2,2,2,0)_{a}$,
$(2,2,2,1,1)_{a}$,
$(2,2,2,1,0)_{a}$,
$(2,2,2,0,0)_{a}$,
$(2,2,1,1,1)_{a}$,
$(2,2,1,1,0)_{a}$,
$(2,2,1,0,0)_{a}$,
$(2,2,0,0,0)_{a}$,
$(2,1,1,1,1)_{a}$,
$(2,1,1,1,0)_{a}$,
$(2,1,1,0,0)_{a}$,
$(2,1,0,0,0)_{a}$,
$(2,0,0,0,0)_{a}$.

As before each of them has four spinors associated.

In the next section we will show that the Pauli terms in the five-dimensional supergravity action allow for mixing of Kaluza-Klein dilatino modes which belong to different mass towers. Thus, also local operators of the form $\mathcal{O}_{k}^{I_{k},(13)}(x)=C_{i_{1} \cdots i_{k}}^{I_{k},(13)} \operatorname{Tr}\left(F_{+}^{2} \bar{\lambda}_{\mathcal{N}=4} X_{i_{1}} \ldots X_{i_{k}}\right)(x)$ give relevant contributions to the structure functions we are interested in. The corresponding irreducible representations of $S U(4)_{R}$ are $[0, k, 1]$. These operators transform in the $(0,1 / 2)$ representation of $S O(3,1)$ and their conformal dimensions are $\Delta=k+11 / 2$. An important point to keep in mind is that for $\mathcal{O}_{k}^{I_{k},(13)}(x)$ operators the relation between the twist and $k$ is now $\tau=k+5$, which is different from the $\mathcal{O}_{k}^{I_{k},(6)}(x)$ operators. Therefore, for $k=0$ which corresponds to twist- 5 spin- $1 / 2$ operators $\mathcal{O}_{k=0}^{I_{0},(13)}(x)$, there are four of such operators which transform in the $\mathbf{4}$ irreducible representation, being this number obtained from the ratio of the values of the Young tableaux:

$$
\begin{array}{|l|}
\hline 4 \\
\hline 3 \\
\hline 2 \\
\hline
\end{array} \text { and } \begin{array}{|l|}
\hline 3 \\
\hline 2 \\
\hline 1 \\
\hline
\end{array}
$$

Next, let us consider the case $k=1$, then $\mathcal{O}_{k=1}^{I_{1},(13)}(x)=$ $C_{i_{1}}^{I_{1},(13)} \operatorname{Tr}\left(F_{+}^{2} \bar{\lambda}_{\mathcal{N}=4} X_{i_{1}}\right)(x)$, corresponding to $\tau=6$, and these operators transform in the $\mathbf{2 0}$ irreducible representation of $S U(4)_{R}$, which is labeled as $[0,1,1]$. The dimension is given by the ratio between the values of these two Young tableaux:

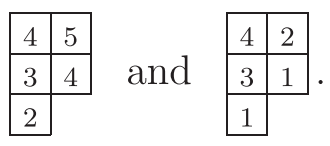

When $k=2$, then $\mathcal{O}_{k=2}^{I_{2},(13)}(x)=C_{i_{1} i_{2}}^{I_{2},(13)} \operatorname{Tr}\left(F_{+}^{2} \bar{\lambda}_{\mathcal{N}=4} X_{i_{1}} X_{i_{2}}\right)(x)$, corresponding to $\tau=7$, and these operators are in the $\mathbf{6 0}$ irreducible representation of $S U(4)_{R}$, which is labeled as 
$[0,2,1]$. The dimension is given by the ratio between the values of the following tableaux:

$$
\begin{array}{|l|l|l|}
\hline 4 & 5 & 6 \\
\hline 3 & 4 & 5 \\
\hline 2 & \multicolumn{1}{|l}{} \\
\cline { 1 - 1 }
\end{array} \text { and } \begin{array}{|l|l|l|}
\hline 5 & 3 & 2 \\
\hline 4 & 2 & 1 \\
\hline 1 & \multicolumn{3}{|c}{} \\
&
\end{array} \text {. }
$$

Thus, we have discussed the identification of the second Dynkin label $k$ of each irreducible representation of $S U(4)_{R}$ with the number $l_{5}$ of the spinor spherical harmonic on $S^{5}$. Another important point that will be specified later is the relation between $l_{1}$ and the charge $\mathcal{Q}$ given in Eq. (3.5).

\section{THE DUAL TYPE IIB SUPERGRAVITY CALCULATION OF THE STRUCTURE FUNCTIONS}

In this section we carry out the holographic dual calculation of the contributions from the single-trace higher-twist spin-1/2 operators to the structure functions. The holographic dual of the large- $N$ limit of $S U(N) \mathcal{N}=4$ SYM theory is given in terms of type IIB supergravity on $\mathrm{AdS}_{5} \times S^{5}$. The metric can be written as

$$
d s^{2}=\frac{d z^{2}+\eta_{\mu \nu} d x^{\mu} d x^{\nu}}{z^{2}}+d \Omega_{5}^{2},
$$

where we set to one radius of $S^{5}$ as well as the scale of the $\mathrm{AdS}_{5}$. The $\mathrm{AdS}_{5}$ indices are $m, n, \ldots=0, \ldots, 4$, the boundary four-dimensional indices are $\mu, \nu, \ldots=0, \ldots, 3$, while the $S^{5}$ indices are $\alpha, \beta, \ldots=1, \ldots, 5$. The bulk coordinate $z \rightarrow 0$ in the UV and we consider a cutoff $z_{0}=1 / \Lambda$ in the IR to induce confinement. This is the so-called hardwall model.

The hadronic tensor can be calculated from the matrix elements of two electromagnetic currents inside the hadron by using the optical theorem. Thus, we have to calculate the imaginary part of the tensor $T^{\mu \nu}$ given in Eq. (1.5) corresponding to the forward Compton scattering. The Gubser-Klebanov-Polyakov-Witten's Ansatz allows us to calculate the above matrix elements by evaluating the onshell supergravity action and taking the sum over all possible intermediate states. Using the covariant type IIB supergravity equations of motion, in [10] we have obtained the effective five-dimensional supergravity action involving two dilatino fields and a massless vector field. We have done it from first principles and therefore we have obtained all the constants from the corresponding angular integrals, which in addition have lead to certain selection rules for the Kaluza-Klein states involved in the fermion interactions. The dilatino field is a right-handed spinor,

$$
\hat{\lambda}(x, y)=\left(\begin{array}{c}
0 \\
\lambda(x, y)
\end{array}\right),
$$

which can be written as a linear combination of the spinor spherical harmonics on $S^{5}$ as

$$
\lambda(x, y)=\sum_{k}\left(\lambda_{k}^{+}(x) \Theta_{k}^{+}(y)+\lambda_{k}^{-}(x) \Theta_{k}^{-}(y)\right),
$$

where $\Theta_{k}^{+}(y)$ and $\Theta_{k}^{-}(y)$ satisfy the Dirac equations on the 5-sphere

$$
\tau^{\alpha} D_{\alpha} \Theta_{k}^{ \pm}=\mp i\left(k+\frac{5}{2}\right) \Theta_{k}^{ \pm} \quad \text { with } \quad k \geq 0
$$

Also, the spinor spherical harmonics turn out to be charge eigenstates satisfying

$$
\left(v^{\alpha} D_{\alpha}-\frac{1}{4} \tau^{\alpha} \tau^{\gamma} \nabla_{\gamma} v_{\alpha}\right) \Theta_{k}^{ \pm}=-i \mathcal{Q} \Theta_{k}^{ \pm}
$$

$\lambda_{k}^{ \pm}$are Kaluza-Klein fields with masses given by $m_{k}^{ \pm}$ defined on the $\mathrm{AdS}_{5}$, while the superscripts \pm indicate the two towers of masses associated with the irreducible representations $\mathbf{4}^{*}, \mathbf{2 0} \mathbf{0}^{*}, \mathbf{6 0}, \ldots(-)$, or $\mathbf{4}, \mathbf{2 0}, \mathbf{6 0}, \ldots(+)$ of the $S O(6) \sim S U(4)$ isometry group. Coordinates $x$ and $y$ are on $\mathrm{AdS}_{5}$ and on $S^{5}$, respectively. Gamma matrices in $\mathrm{AdS}_{5}$ and $S^{5}$ are denoted by $\gamma^{m}$ and $\tau^{\alpha}$, respectively. They satisfy the Clifford algebra

$$
\left\{\gamma_{\hat{a}}, \gamma_{\hat{b}}\right\}=2 \eta_{\hat{a} \hat{b}}, \quad\left\{\tau_{\hat{\alpha}}, \tau_{\hat{\beta}}\right\}=2 \delta_{\hat{\alpha} \hat{\beta}},
$$

where indices $\hat{a}, \hat{b}, \hat{c}, \ldots$ and $\hat{\alpha}, \hat{\beta}, \hat{\gamma}, \ldots$ correspond to flat space-time. The vielbein field $e_{a}^{\hat{b}}$ is used to relate the $\operatorname{AdS}$ indices to flat-space indices. Analogously, $e_{\alpha}^{\hat{\beta}}$ is associated with the $S^{5}$.

The structure functions of polarized spin- $1 / 2$ hadrons related to operators of the type $\mathcal{O}_{k}^{I_{k},(6)}$ in the $\mathcal{N}=4 \mathrm{SYM}$ theory can be calculated by using the effective action at leading order obtained in [10]. The following interaction terms have been derived from first principles, i.e., from direct dimensional reduction on $S^{5}$ for the dilatino terms at leading order in type IIB supergravity,

$$
\begin{aligned}
S_{\mathrm{int}}= & K \int d z d^{4} x \sqrt{-g_{\mathrm{AdS}_{5}}} \\
& \times\left(i \frac{\mathcal{Q}}{3} \bar{\lambda}_{k}^{-} \gamma^{a} B_{a}^{1} \lambda_{k}^{-}+i \frac{b_{1 k j}^{-,-}}{12} \bar{\lambda}_{j}^{-} F^{a b} \Sigma_{a b} \lambda_{k}^{-}\right. \\
& \left.+i \frac{b_{1 k j}^{+--}}{12} \bar{\lambda}_{j}^{+} F^{a b} \Sigma_{a b} \lambda_{k}^{-}\right)
\end{aligned}
$$

where 


$$
\begin{aligned}
b_{1 k j}^{ \pm,-}= & \left(1+2\left(k \mp j+\frac{5}{2} \mp \frac{5}{2}\right)\right) \int d \Omega_{5}\left(\Theta_{j}^{ \pm}\right)^{\dagger} \tau_{\alpha} v^{\alpha} \Theta_{k} \\
& +4 \mathcal{Q} \int d \Omega_{5}\left(\Theta_{j}^{ \pm}\right)^{\dagger} \Theta_{k}^{-} .
\end{aligned}
$$

$K$ is a normalization constant that can be calculated by comparison with the type IIB supergravity action of Ref. [42]. In addition, $B_{a}^{1}$ is the massless Maxwell-Einstein field in $\mathrm{AdS}_{5}$ defined in Eq. (3.9), while $F_{a b}=\nabla_{a} B_{b}^{1}-$ $\nabla_{b} B_{a}^{1}$ and $\Sigma_{a b}=\frac{1}{4}\left(\gamma_{a} \gamma_{b}-\gamma_{b} \gamma_{a}\right)$. Early references for the covariant equations of motions of type IIB supergravity fields are [43-45].

The first term in the action (3.7) corresponds to the minimal-coupling interaction used to calculate the structure functions of a DIS process in [4,5] and the vector-spinorspinor three-point function in Ref. [46]. This coupling only connects states in the same irreducible representation (which have the same twist). The other interactions are Pauli terms whose strengths are given by the coefficients $b_{1 k j}$ calculated from the angular integrals of spinor spherical harmonics. We can separate contributions having states in the same irreducible representation, and mixing of states from different irreducible representations of $S U(4)$, which are given by the second and the third terms, respectively.

Before studying the selection rules for higher-twist operators, we briefly review the solutions in $\mathrm{AdS}_{5}$ of non-normalizable modes of the vector field and the normalizable modes of dilatini, which correspond to the holographic dual fields of the electromagnetic current and the hadronic states, respectively. These solutions will be inserted in the effective five-dimensional action (3.7) to calculate the matrix elements of the electromagnetic currents.

The massless vector fields $B_{a}^{1}$ come from a certain linear combination of off-diagonal fluctuations of the metric tensor and vector fluctuations of the Ramond-Ramond four-field potential in the following way:

$$
B_{a}^{1}(x) \equiv A_{a}^{1}(x)-16 \Phi_{a}^{1}(x),
$$

where $A_{a}^{1}(x)$ is defined by the metric fluctuation as

$$
h_{a \alpha}=\sum_{I_{5}} A_{a}^{I_{5}}(x) Y_{\alpha}^{I_{5}}(y),
$$

and $\Phi_{a}^{1}(x)$ is given in terms of the mode expansion of the Ramond-Ramond field as

$$
a_{a \alpha \beta \gamma}=\sum_{I_{5}} \Phi_{a}^{I_{5}}(x) \epsilon_{\alpha \beta \gamma \delta \epsilon} \nabla^{\delta} Y^{I_{5} \epsilon}(y)
$$

In particular, the index $I_{5}$ denotes the set of numbers $\left(l_{5}, l_{4}, l_{3}, l_{2}, l_{1}\right)$ for the vector spherical harmonics on $S^{5}$, $Y^{I_{5} \epsilon}(y)$. The corresponding masses of these vector fields are given by $M_{B, l}^{2}=l^{2}-1$ with $l \geq 1$, therefore they only depend on $l \equiv l_{5}$ and, in terms of the irreducible representations of $S U(4)$ they transform in the $\mathbf{1 5}, \mathbf{6 4}, \mathbf{1 7 5}, \ldots$, for $l=1,2,3, \ldots$. For the holographic DIS calculation we only need to consider the massless vector fields, i.e., $B_{a}^{1}(x)$, which are the 15 Yang-Mills fields of $S U(4) \sim S O(6)$. In addition, in this case the vector spherical harmonics are Killing vectors of $S^{5}$. The gauge fields satisfy the following Einstein-Maxwell equation of motions in $\mathrm{AdS}_{5}$ :

$$
\begin{gathered}
\nabla_{a} F^{a b}=0, \\
\partial^{\mu} B_{\mu}^{1}+z \partial_{z}\left(\frac{B_{z}^{1}}{z}\right)=0 .
\end{gathered}
$$

The second equation gives a Lorentz-type gauge fixing condition. Then, the non-normalizable modes which are dual to the hadronic current on the boundary satisfy the following boundary condition:

$$
B_{\mu}^{1}\left(x_{\nu}, z \rightarrow 0\right)=n_{\mu} e^{i q \cdot x} .
$$

Thus, the solutions of Eqs. (3.12) and (3.13) with the boundary condition (3.14) read

$$
\begin{aligned}
& B_{\mu}^{1}\left(x_{\nu}, z\right)=n_{\mu} e^{i q \cdot x} q z K_{1}(q z), \\
& B_{z}^{1}\left(x_{\nu}, z\right)=i n \cdot q e^{i q \cdot x} z K_{0}(q z) .
\end{aligned}
$$

On the other hand, the dilatini satisfy the Dirac equation in $\mathrm{AdS}_{5}$ with the hard-wall boundary condition at the IR, needed in order to break the conformal symmetry and induce color confinement. Thus, we impose Dirichlet boundary conditions at the IR cutoff $z_{0}=1 / \Lambda$. In addition, in the ultraviolet region $(z \rightarrow 0)$ the boundary condition is fixed by choosing the normalizable mode for the initial and final hadronic states. The Dirac equation in $\mathrm{AdS}_{5}$ reads

$$
\left(z \gamma^{m} \partial_{m}-2 \gamma^{5}-k-\frac{3}{2}\right) \lambda_{k}^{-}=0,
$$

being the normalizable solution

$$
\begin{aligned}
& \lambda_{k}^{-}\left(x_{\nu}, z\right) \\
& \quad=C e^{i P \cdot x} z^{\frac{5}{2}}\left(J_{\tau-2}(M z) P_{+}+J_{\tau-1}(M z) P_{-}\right) u_{\sigma},
\end{aligned}
$$

where the projectors are

$$
P_{ \pm}=\frac{\left(I \pm \gamma^{5}\right)}{2},
$$

while $P^{\mu}$ is the four-momentum of the hadron, and the solution has been expressed in terms of Bessel functions of the first kind and four-dimensional Dirac spinors $u_{\sigma}$. These spinors satisfy $\gamma^{\mu} P_{\mu} u_{\sigma}=i M u_{\sigma}$ with $P^{2}=-M^{2}$. The twist 
$\tau=\Delta-\frac{1}{2}=m_{k}+3 / 2$ corresponds to the $\mathcal{N}=4 \mathrm{SYM}$ operator $\mathcal{O}_{k}^{I_{k},(6)}$. The constant $C=c_{\mathcal{X}}^{\prime} M_{\mathcal{X}}^{1 / 2} / z_{0}^{1 / 2}$ can be expressed in terms of another dimensionless constant $c_{\mathcal{X}}^{\prime}$, following the normalization used in Ref. [4]. The identity matrix is indicated as $I$. In addition, the bulk solutions for the fields $\lambda^{+}$dual to $\mathcal{O}_{k}^{I_{k},(13)}$ operators are calculated in the same way.

The coefficients in the effective action (3.7) are given in terms of integrals of spinor spherical harmonics on $S^{5}$, and they lead to the selection rules for the intermediate states in the forward Compton scattering. Recall that the spinor spherical harmonics have five quantum numbers $\left(l_{5}, l_{4}, l_{3}, l_{2}, l_{1}\right)_{a}$, which satisfy the conditions $l_{5} \geq l_{4} \geq l_{3} \geq l_{2} \geq l_{1} \geq 0$. We also use the subscripts $a=1,2,3,4$. In particular, $l_{5}=k$ is related to the twist, while the $l_{1}$ index is associated with the charge $\mathcal{Q}= \pm\left(l_{1}+\frac{1}{2}\right)$. For $k=0$, from the dual SYM theory point of view, the operators $\mathcal{O}_{k=0}^{I_{0},(6)}$ belong to the $\mathbf{4}^{*}$ irreducible representation of $S U(4)_{R}$. Thus, there are four of these type of operators, and we have explicitly verified that the final result for the structure functions is the same for all these operators belonging to the $4^{*}$ representation. Similarly, for $k=1$ there are 20 Kaluza-Klein states (and operators) which can be separated in five sets, leading to the same structure functions within each set. An analogous situation occurs for $k=2$ where from the 60 Kaluza-Klein states (and operators) there are 15 different sets with the same structure functions for the four states within the same set. We have checked these results.

The minimal coupling only connects states with the same quantum numbers $\left(l_{5}, l_{4}, l_{3}, l_{2}, l_{1}\right)_{a}$ on $S^{5}$ and belong to the same irreducible representation of $S U(4) \sim S O(6)$. The contributions to the structure functions associated with the minimal coupling are denoted by $F_{i}^{m}$ and $g_{i}^{m}$. They have been calculated in [10] and their explicit dependence in $q$ and $x$ is detailed in Appendix A. The other terms of the effective action (3.7) are Pauli interactions with coefficients $b_{1 k j}$. In the second term we can identify the interaction between the gauge field and two dilatini of the same Kaluza-Klein mass tower. Therefore, they correspond to operators in the same irreducible representation of $S U(4)_{R}$. The angular integrals only connect states with equal twist. The matrix elements can be calculated by solving the $z$-integrals on $\mathrm{AdS}_{5}$. In Appendix A these functions are also written in detail, and we denote them by a superscript $P$, namely: $F_{i}^{P}$ and $g_{i}^{P}$. These interactions can be separated into two sets. The first set is constituted by diagrams which have intermediate states with the same quantum numbers $\left(l_{5}, l_{4}, l_{3}, l_{2}, l_{1}\right)_{a}$ as the incident dilatino. Thus, we can calculate their coefficients by the following integral:

$$
b_{1 k k}^{-,-}=(11+4 k) \int d \Omega_{5}\left(\Theta_{k}^{-}\right) \dagger \tau_{\alpha} v^{\alpha} \Theta_{k}^{-}+4 \mathcal{Q} .
$$

We define the constant

$$
\beta_{P}=\frac{b_{1 k k}^{-,-}}{12} .
$$

This Pauli interaction term is particularly important since when we calculate the product of the one-point function of the electromagnetic current and its complex conjugate in Eq. (1.5), there is a cross contribution corresponding to a Feynman-Witten diagram which also includes the minimal coupling. This leads to matrix elements of the hadronic tensor of the form

$$
\begin{aligned}
n_{\mu} n_{\nu} W^{c \mu \nu}= & n_{\mu} n_{\nu} 2 \pi^{2} \sum_{\mathcal{X}} \delta\left(M_{\mathcal{X}}^{2}+(P+q)^{2}\right)\left(\left\langle J_{m}^{\mu}(0)\right\rangle\left\langle J_{P}^{* \nu}(0)\right\rangle\right. \\
& \left.+\left\langle J_{P}^{\mu}(0)\right\rangle\left\langle J_{m}^{* \nu}(0)\right\rangle\right) .
\end{aligned}
$$

The structure functions from cross terms are indicated with the superscript $c: F_{i}^{c}$ and $g_{i}^{c}$ and they are explicitly shown in Appendix A.

In Fig. 1 we illustrate the Feynman-Witten diagrams needed to calculate the matrix elements of the electromagnetic currents inside the hadron. The first matrix elements correspond to the same incoming and outgoing state, with the minimal coupling and the Pauli term (dotted vertex) discussed above. On the other hand, as already mentioned the Pauli diagrams with final states belonging to same irreducible representations of $S U(4)$ (i.e., equal $k=l_{5}$ ), but different $l_{4}, l_{3}, l_{2}, l_{1}$ numbers, give the same contributions to the structure functions. However, they do not lead to cross terms involving the minimal coupling. Now, we define the following constant:

$$
\begin{aligned}
\beta_{P m}^{2} & =\sum_{I_{5}}\left(\frac{b_{1 k k}^{--}}{12}\right)^{2} \\
& =\sum_{I_{5}}\left(\frac{(11+4 k) \int d \Omega_{5}\left(\Theta_{k}^{+}\right)^{\dagger} \tau_{\alpha} v^{\alpha} \Theta_{k}^{-}}{12}\right)^{2},
\end{aligned}
$$

where $I_{5}$ indicates the quantum numbers $\left(l_{5}, l_{4}, l_{3}, l_{2}, l_{1}\right)_{a}$ of the possible intermediate states.

The last term of Eq. (3.7) couples fermionic modes of different Kaluza-Klein towers of type IIB supergravity compactified on $S^{5}$. Considering Feynman-Witten diagrams with incoming states dual to the operators $\mathcal{O}_{k}^{I_{k},(6)}$, the intermediate states will be dual to $\mathcal{O}_{k^{\prime}}^{I_{k^{\prime}},(13)}$ with $k^{\prime}=k+1$ or $k^{\prime}=k-1$. The selection rule $k^{\prime}=k \pm 1$ is obtained from the angular integral of the spinor spherical harmonics, ${ }^{2}$ being the matrix elements calculated using the Feynman-Witten diagrams of the second and third lines in Fig. 1.

\footnotetext{
${ }^{2}$ These selection rules relate the conformal dimensions of the incident and intermediate states through $\Delta_{\mathcal{X}}=\Delta_{i}+2 \pm 1$.
} 


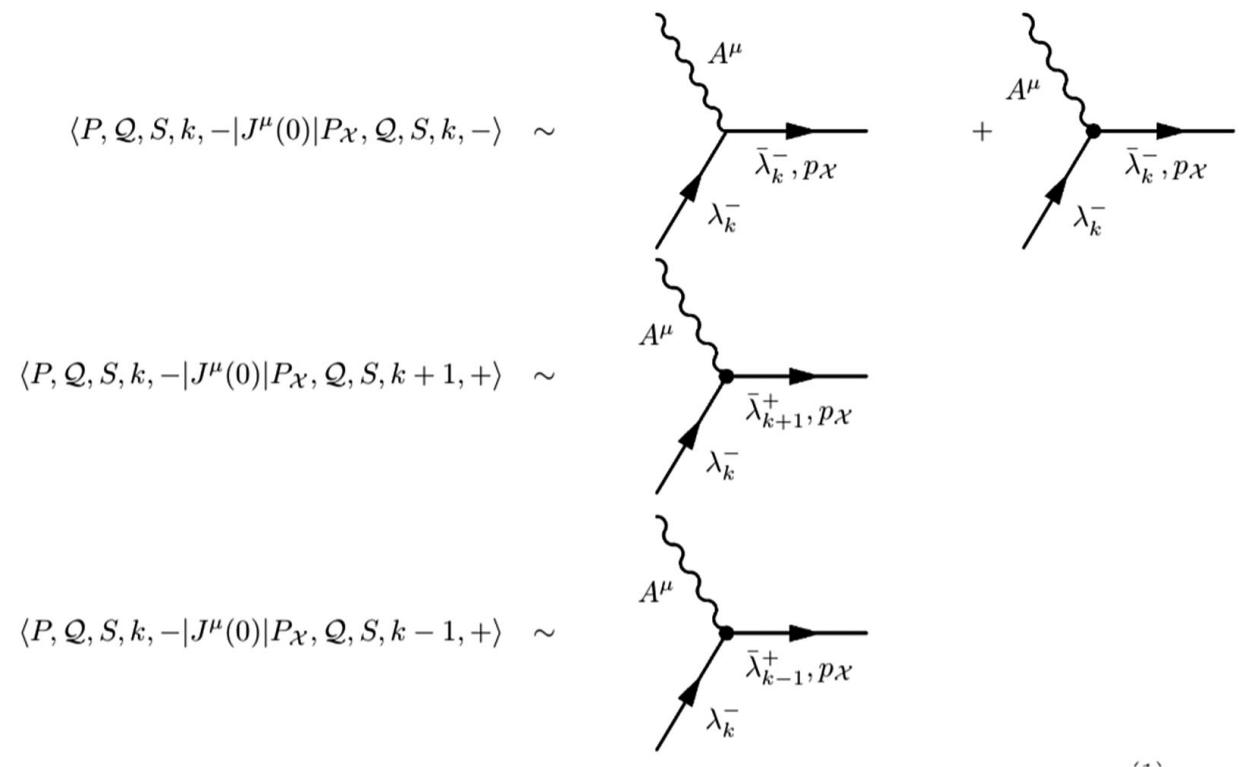

FIG. 1. Matrix elements of the electromagnetic current inside the hadron are written on the left. The minimal coupling is represented by the first diagram. The diagrams with dotted vertices correspond to different Pauli terms. Selection rules allow for these three Pauli diagrams.

The constant $\beta_{-}^{2}$ for the case $k^{\prime}=k-1$ is calculated in terms of the coefficients $b_{1 k j}^{+,-}$of the effective action as follows:

$$
\beta_{-}^{2}=\sum_{I_{5}}\left(\frac{b_{1 k k-1}^{+,-}}{12}\right)^{2}=\sum_{I_{5}}\left(\frac{\int d \Omega_{5}\left(\Theta_{k-1}^{+}\right)^{\dagger} \tau_{\alpha} v^{\alpha} \Theta_{k}^{-}}{4}\right)^{2} .
$$

The corresponding contributions to the structure functions are given by

$$
\begin{gathered}
F_{1}^{P-}=\frac{F_{3}^{P-}}{2}=g_{1}^{P-}=\frac{g_{5}^{P-}}{2}=\frac{1}{2}\left|a_{0}\right|^{2}\left(\frac{\Lambda^{2}}{q^{2}}\right)^{\tau-1}(1-x)^{\tau-2} x^{\tau+1} \Gamma(\tau)^{2}, \\
F_{2}^{P-}=g_{4}^{P-}=\left|a_{0}\right|^{2}\left(\frac{\Lambda^{2}}{q^{2}}\right)^{\tau-1}(1-x)^{\tau-3} x^{\tau+1} \Gamma(\tau)^{2}(1+x(3+\tau(-2+x(\tau-2)))), \\
g_{2}^{P-}=-\frac{1}{4}\left|a_{0}\right|^{2}\left(\frac{\Lambda^{2}}{q^{2}}\right)^{\tau-1} \frac{\Gamma(\tau)^{2}}{\tau-1} x^{\tau+1}(1-x)^{\tau-3}\left[-\tau-1+x\left(x^{5}(\tau+1) \tau(\tau-2)+(1+\tau)^{2}\right.\right. \\
-x^{4}(\tau-2) \tau(7+3 \tau)+6 x^{2}(2+(3-2 \tau) \tau) \\
\left.\left.-x(12+(\tau-5) \tau)+x^{3}(\tau-3)(2+3 \tau(5+\tau))\right)\right], \\
g_{3}^{P-}=\left|a_{0}\right|^{2}\left(\frac{\Lambda^{2}}{q^{2}}\right)^{\tau-1} \frac{\Gamma(\tau)^{2}}{\tau-1} x^{\tau+1}(1-x)^{\tau-3} \\
\quad \times(3-\tau+x(-15-4(x-3) x-2 \tau+x(33-2 x(18+(x-8) x)) \tau \\
\left.\left.+(1+x(-1+(x-6) x(3+x(x-3)))) \tau^{2}+x^{3}\left(3+x(x-3) \tau^{3}\right)\right)\right),
\end{gathered}
$$

where the constant $a_{0}=2 \pi c_{i}^{\prime} c_{\mathcal{X}}^{\prime} 2^{\tau} K$ is written in terms of the factors $c_{i}^{\prime}$ and $c_{\mathcal{X}}^{\prime}$ corresponding to the normalization constants of the incident and the intermediate hadronic wave functions, respectively. We would like to emphasize that this interaction is not allowed for the case of $\tau=3$ studied in [10]. For this reason it is interesting to study its role for highertwist operators. Finally, we consider the case $k^{\prime}=k+1$, with a constant given by 


$$
\beta_{+}^{2}=\sum_{I_{5}}\left(\frac{b_{1 k k+1}^{+,-}}{12}\right)^{2}=\sum_{I_{5}}\left(-\frac{\int d \Omega_{5}\left(\Theta_{k+1}^{+}\right)^{\dagger} \tau_{\alpha} v^{\alpha} \Theta_{k}^{-}}{12}\right)^{2} .
$$

The structure functions associated with these interactions have different dependence on the Bjorken parameter,

$$
\begin{aligned}
F_{1}^{P+}= & \frac{F_{3}^{P+}}{2}=g_{1}^{P+}=\frac{g_{5}^{P+}}{2} \\
= & \frac{1}{2}\left|a_{0}\right|^{2}\left(\frac{\Lambda^{2}}{q^{2}}\right)^{\tau-1} x^{-\tau-3}(1-x)^{\tau+2} \Gamma(1+\tau)^{2} \\
& \times\left[x^{\tau+1}-(1+\tau)_{2} F_{1}\left(\tau+2, \tau+2, \tau+3, \frac{x-1}{x}\right)\right]^{2}, \\
F_{2}^{P+}= & g_{4}^{P+} \\
= & \left|a_{0}\right|^{2}\left(\frac{\Lambda^{2}}{q^{2}}\right)^{\tau-1} x^{-\tau}(1-x)^{\tau+1} \Gamma(1+\tau)^{2} \\
& \times\left[x^{2 \tau}+\frac{1-x}{x^{3}}(1+\tau)^{2}{ }_{2} F_{1}\left(\tau+2, \tau+2, \tau+3, \frac{x-1}{x}\right)^{2}\right], \\
g_{2}^{P+}=- & \frac{1}{4}\left|a_{0}\right|^{2}\left(\frac{\Lambda^{2}}{q^{2}}\right)^{\tau-1}(1-x)^{\tau} x^{\tau} \Gamma(1+\tau)^{2} \\
\times & {\left[2 \frac{1-x}{1-\tau}+(1-x)^{-2 \tau}(1+\tau)^{2}(2+\tau) B\left(\frac{x-1}{x}, \tau+2,-(\tau+1)\right)\right.} \\
\times & \left(\frac{(x-1)^{\tau}}{1-\tau}+\frac{x(\tau+2) B\left(\frac{x-1}{x}, \tau+2,-(\tau+1)\right)}{(1-x)^{2}}\right), \\
g_{3}^{P+}= & \left|a_{0}\right|^{2}\left(\frac{\Lambda^{2}}{q^{2}}\right)^{\tau-1} \frac{1}{\tau-1} 8(1-x)^{\tau+1} x^{-\tau-3} \Gamma(1+\tau)^{2} \\
\times & {\left[2 x^{2 \tau+3}+(1+\tau)^{2}(x-1){ }_{2} F_{1}\left(\tau+2, \tau+2, \tau+3, \frac{x-1}{x}\right)\right.} \\
\times & \left(-x^{\tau+1}+(\tau-1){ }_{2} F_{1}\left(\tau+2, \tau+2, \tau+3, \frac{x-1}{x}\right)\right),
\end{aligned}
$$

which are expressed in terms of the incomplete Beta function, $B(x, a, b)$, and the hypergeometric function, ${ }_{2} F(a, b, c, x)$.

The general form of the structure functions which contain all the contributions for higher-twist operators is given by

$$
\begin{aligned}
F_{i}= & \beta_{m}^{2} F_{i}^{m}+\beta_{P}^{2} F_{i}^{P}+\beta_{m} \beta_{P} F_{i}^{c}+\beta_{P m}^{2} F_{i}^{P} \\
& +\beta_{+}^{2} F_{i}^{P+}+\beta_{-}^{2} F_{i}^{P-},
\end{aligned}
$$

and we have a similar expression for the $g_{i}$ structure functions. For the complete structure functions, i.e., by adding all the contributions from all allowed interactions from action (3.7), for any twist, there are the following relations:

$$
F_{1}=\frac{F_{3}}{2}=g_{1}=\frac{g_{5}}{2}, \quad \text { and } \quad F_{2}=g_{4} .
$$

Thus, in what follows we will show explicitly $F_{1}, F_{2}, g_{2}$ and $g_{3}$, which are independent.

For $\tau=3$ analyzed in [10], the constants $\beta_{-}^{2}$ and $\beta_{P m}^{2}$ vanish since the incident hadron has $k=l_{5}=0$ and, consequently all the remaining $l_{i}$ 's are zero. However, for higher-twist operators these contributions are nonzero. In the following subsection we will analyze how each interaction contributes to the structure functions for $\tau=4$ and $\tau=5$. 


\section{A. The case of twist-4 $\mathcal{O}_{k=1}^{I_{1},(6)}=C_{i_{1}}^{I_{1},(6)} \operatorname{Tr}\left(F_{+} \lambda_{\mathcal{N}=4} X_{i_{1}}\right)$ operators}

In this case the incident hadrons correspond to operators of the form $\mathcal{O}_{k=1}^{I_{1},(6)}$ which belong to the $\mathbf{2 0}^{*}$ irreducible representation of $S U(4)_{R}$. They have twist $\tau=4$. The holographic dual fields are represented by dilatino modes with $l_{5}=k=1$ and their respective quantum numbers are $I_{5}=\left(1, l_{4}, l_{3}, l_{2}, l_{1}\right)_{a}$.

There are 20 independent spinor spherical harmonics with the same Kaluza-Klein mass. This degeneration comes from the possibility of having $I_{5}=\left(1, l_{4}, l_{3}, l_{2}, l_{1}\right)_{a}$ values satisfying $l_{5}=1 \geq l_{4} \geq l_{3} \geq l_{2} \geq l_{1} \geq 0$ (in fact there are five combinations) times the 4 degrees of freedom of each spinor, which are parametrized by the subscripts $a=1, \ldots, 4$. The final result only depends on the choice of the $l_{i}$ 's, thus we can separate the 20 initial possibilities in five sets as commented before. Once we choose a certain set of $l_{i}$ 's, the four possible states lead to the same structure functions. Therefore, without loss of generality we choose $a=1$ for the incident hadron. For instance, the spinor spherical harmonic normalized with $I_{5}=(1,0,0,0,0)_{a=1}$ is

$$
\Theta_{(1,0,0,0,0)_{a=1}}^{-}=\frac{e^{-i \mathcal{Q} \theta_{1}}}{\sqrt{5} \pi^{3 / 2}}\left[\begin{array}{c}
e^{-i \frac{1}{2}\left(\theta_{3}-\theta_{5}\right)} \cos \left(\frac{\theta_{2}}{2}\right) \cos \left(\frac{\theta_{4}}{2}\right)\left(5 i \cos \left(\theta_{5}\right)-\sin \left(\theta_{5}\right)\right) \\
-e^{i \frac{1}{2}\left(\theta_{3}+\theta_{5}\right)} \sin \left(\frac{\theta_{2}}{2}\right) \cos \left(\frac{\theta_{4}}{2}\right)\left(5 i \cos \left(\theta_{5}\right)-\sin \left(\theta_{5}\right)\right) \\
-e^{-i \frac{1}{2}\left(\theta_{3}+\theta_{5}\right)} \cos \left(\frac{\theta_{2}}{2}\right) \sin \left(\frac{\theta_{4}}{2}\right)\left(5 i \cos \left(\theta_{5}\right)+\sin \left(\theta_{5}\right)\right) \\
e^{-i \frac{1}{2}\left(-\theta_{3}+\theta_{5}\right)} \sin \left(\frac{\theta_{2}}{2}\right) \sin \left(\frac{\theta_{4}}{2}\right)\left(5 i \cos \left(\theta_{5}\right)+\sin \left(\theta_{5}\right)\right)
\end{array}\right] .
$$

As mentioned, the angular integrals of the spinor spherical harmonics allow us to obtain the selection rules and the relative coefficients among the contributions given by different terms in the action (3.7). There is only one state with $l_{1}=1$, i.e., with $\mathcal{Q}= \pm \frac{3}{2}$, while the other four have $l_{1}=0$, i.e., with $\mathcal{Q}= \pm \frac{1}{2}$. This is important because the charge is a conserved quantity and the integrals are equal to zero if we mix states with different $l_{1}$. Since we have considered an incident state with $a=1$, the only possibility is the coupling with states with $a=1$ and 3, because they have the same charge. Spinors with $a=2,4$ have charge with a different sign. Tables collecting the details of intermediate states and their coefficients obtained from the angular integrals of the spinor spherical harmonics are displayed in Appendix B. There we note that the selection rules for $l_{i}$, with $i=2,3,4$, are given by $l_{i}^{\prime}=l_{i} \pm 1$ as long as they satisfy $l_{5} \geq l_{4} \geq l_{3} \geq l_{2} \geq l_{1}$.

The twists of the incident and the intermediate states, related to the values of $l_{5}=l=k$, set the dependence on the Bjorken variable as well as on the virtual-photon momentum transfer. Then, the degeneracy given by the rest of numbers $l_{4}, l_{3}, l_{2}$ and $l_{1}$ can enhance the relative coefficient of a given contribution. In Figs. 2 and 3 we draw the structure functions $F_{1}, F_{2}$ and $g_{2}$ for the five possible incident hadrons with $\left(1, l_{4}, l_{3}, l_{2}, l_{1}\right)_{a}$ numbers and $\tau=4$. We have set $\left|a_{0}\right|=1$ which is the only free constant for all the structure functions. In addition we have factorized out $\left(\Lambda^{2} / q^{2}\right)^{\tau-1}$. In each subfigure the different contributions of Eq. (3.33) are displayed with different colors (see figure captions), while blue lines indicate the full structure functions (including all possible contributions). The corresponding curves for $g_{3}$ are displayed in Appendix C.
First, we note that for almost all the structure functions, the minimal coupling contributions (orange line) are very small in comparison with the rest of the contributions. This effect has been observed in [10] for $\tau=3$. However, in the case with maximum charge $\mathcal{Q}=3 / 2\left(l_{1}=1\right)$ they have a similar magnitude in comparison with the other terms as shown in Fig. 3. Second, with respect to the Pauli terms, let us consider the coupling which connects states belonging to the same $2 \mathbf{2 0}^{*}$ irreducible representation of $S U(4)$ (green and violet lines). They show a bell-shaped form with maximum near $x \sim 0.75$, and also they fall off as $(1-x)^{\tau-2}$ as $x \rightarrow 1$. These terms are very important for the structure functions with $\mathcal{Q}=1 / 2$, even taking into account the suppression of the red line given by the cross terms. The behavior of the violet line is controlled by the set of states $\left(1, l_{4}, l_{3}, l_{2}, 0\right)$ (see Fig. 2), and they do not contribute if the incident hadron has charge $\mathcal{Q}=3 / 2$ (Fig. 3).

On the other hand, there are also contributions from the diagrams with intermediate states associated with operators $\mathcal{O}_{k=0}^{I_{0},(13)}$ which belong to the $\mathbf{4}$ irreducible representation of $S U(4)_{R}$. The case corresponding to the selection rule $k^{\prime}=k+1$ (indicated in the figures with brown lines) is interesting since it is relevant for all the structure functions. Particularly, for $\mathcal{Q}=3 / 2$, this represents the main contribution at relatively low $x$. The corresponding curves have maxima around $x \sim 0.35$ and fall off rapidly for higher values of the Bjorken parameter. Finally, the light-blue curves correspond to the case when $k^{\prime}=k-1$, and they display their maxima around $x \sim 0.9$. For $\mathcal{Q}=3 / 2$ charge conservation does not allow for this type of coupling, thus the coefficients obtained from the 


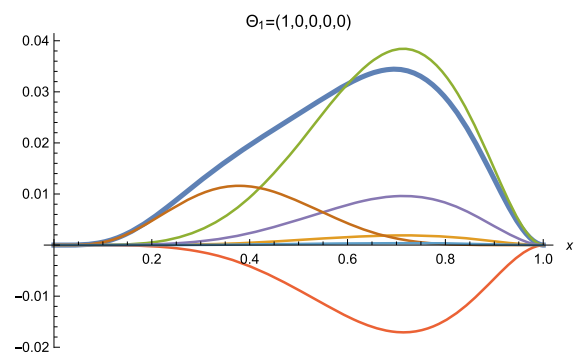

(a) $F_{1}$

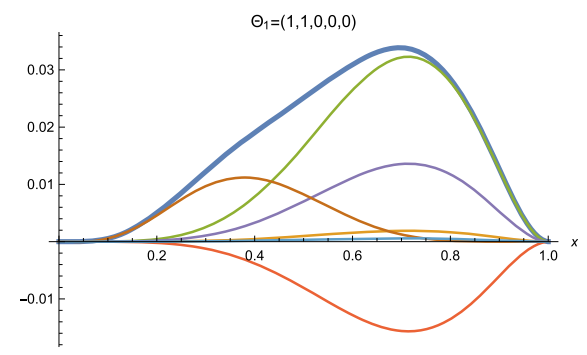

(d) $F_{1}$

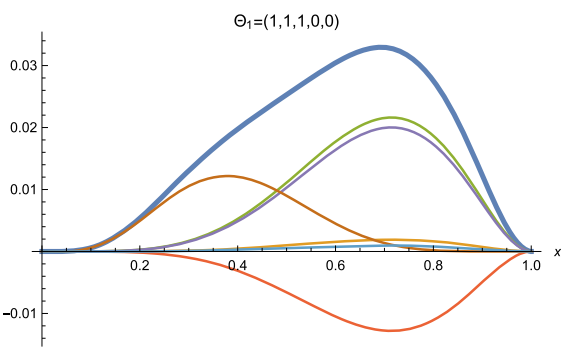

(g) $F_{1}$

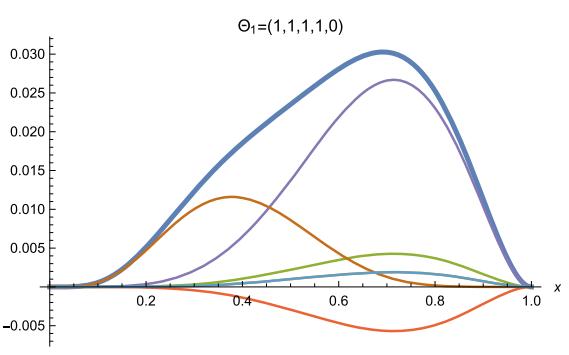

(j) $F_{1}$

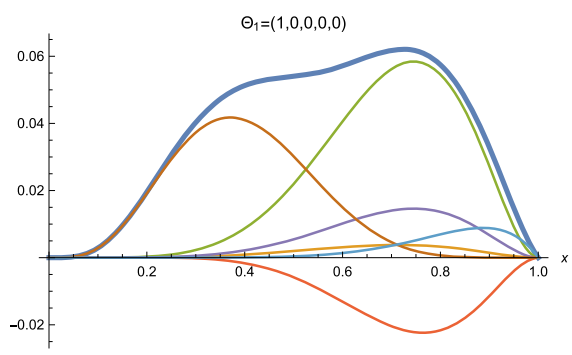

(b) $F_{2}$

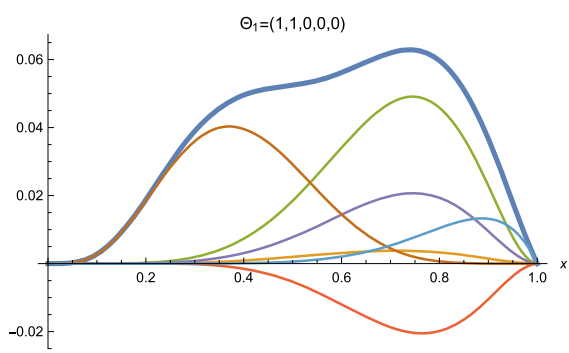

(e) $F_{2}$

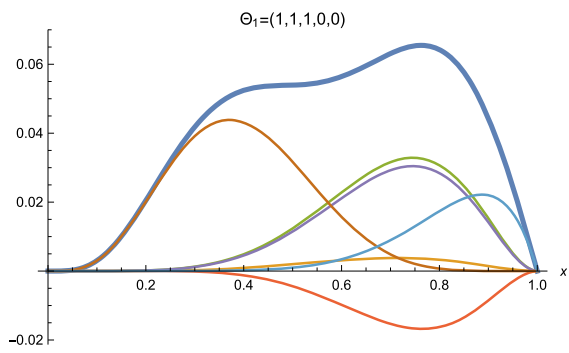

(h) $F_{2}$

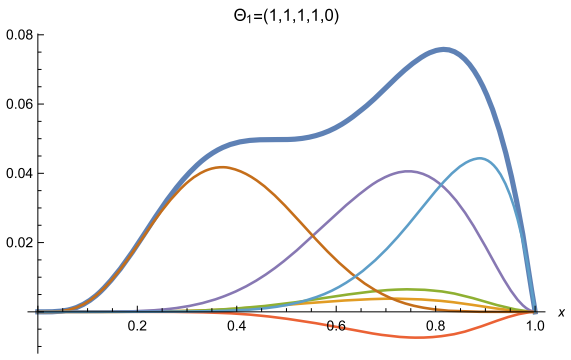

(k) $F_{2}$

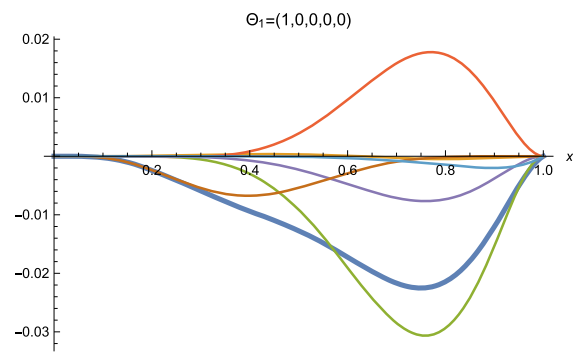

(c) $g_{2}$

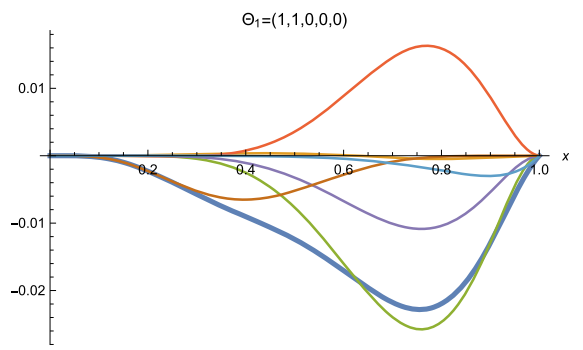

(f) $g_{2}$

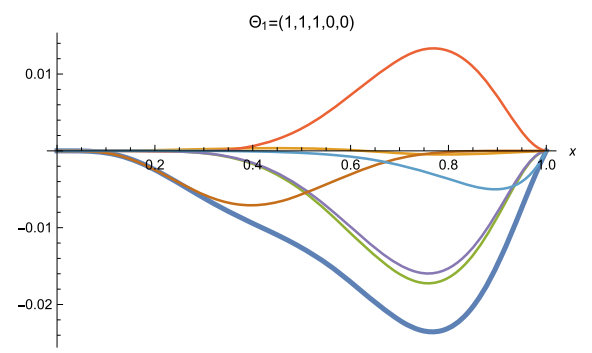

(i) $g_{2}$

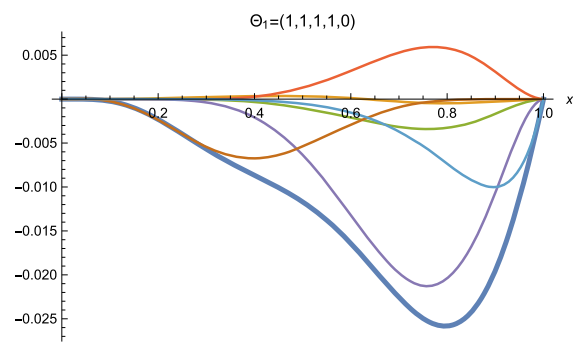

(l) $g_{2}$

FIG. 2. Full structure functions $F_{1}, F_{2}$ and $g_{2}$ (blue line) as functions of the Bjorken parameter $x$, obtained from twist-4 spin- $1 / 2$ fermionic operators $\mathcal{O}_{k=1}^{I_{1},(6)}$ with $l_{5}=1 \geq l_{4} \geq l_{3} \geq l_{2} \geq l_{1}=0(\mathcal{Q}=1 / 2)$. We also display all the contributions coming from different terms, namely: from the minimal coupling $\beta_{m}^{2} F_{1}^{m}$ (orange line); the Pauli interaction where the intermediate state $\lambda_{\mathcal{X}} \equiv \lambda_{k=1}^{-}$is the same as the incident state $\beta_{P}^{2} F_{1}^{P}$ (green line), and where the intermediate state is different $\beta_{P m}^{2} F_{1}^{P}$ (violet line), both belonging to the 20*; the contribution from cross terms $\beta_{m} \beta_{P} F_{1}^{c}$ (red line); and the contributions from the Pauli interactions with intermediate states in the 60: $\beta_{+}^{2} F_{1}^{P+}$, where $\lambda_{\mathcal{X}} \equiv \lambda_{k+1}^{+}$(brown line); and in the 4: $\beta_{-}^{2} F_{1}^{P-}$, where $\lambda_{\mathcal{X}} \equiv \lambda_{k-1}^{+}$(light blue line).

spinor-spherical-harmonics angular integrals vanish in this case. For $\mathcal{Q}=1 / 2$ these couplings become negligible for $F_{1}$, being only relevant for incident hadrons with quantum numbers $(1,1,1,0,0)$ and $(1,1,1,1,0)$ for $F_{2}$ and $g_{2}$.
Figure 4 shows the full structure functions for each possible initial state with $\tau=4$. The curves with the same charge $(\mathcal{Q}=1 / 2)$ have similar bell-shaped curves. The region of $0.6 \leq x<1$ is dominated by contributions 


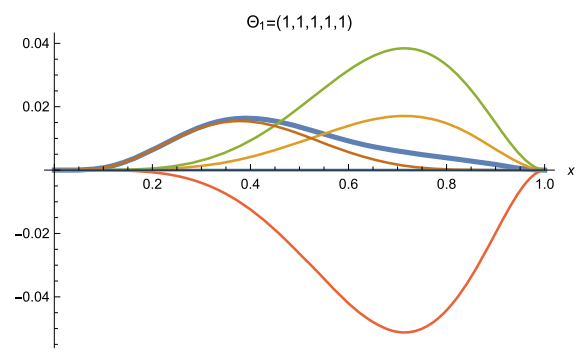

(a) $F_{1}$

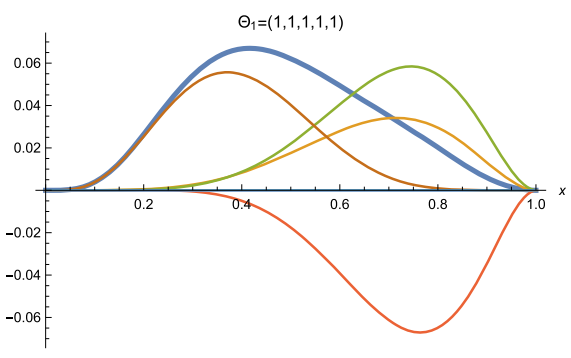

(b) $F_{2}$

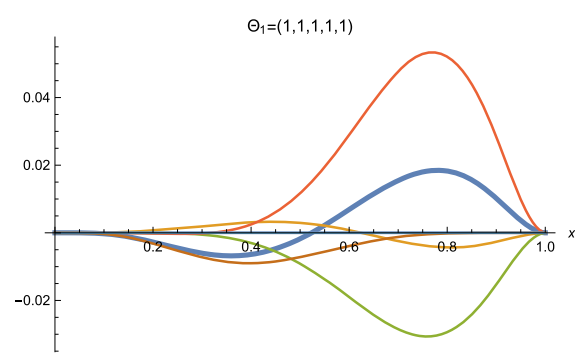

(c) $g_{2}$

FIG. 3. Full structure functions $F_{1}, F_{2}$ and $g_{2}$ (blue line) as functions of the Bjorken parameter $x$ and the detail of each contributions, obtained from the twist- 4 spin- $1 / 2$ fermionic operators $\mathcal{O}_{k=1}^{I_{1},(6)}$ with $l_{5}=l_{4}=l_{3}=l_{2}=l_{1}=1(\mathcal{Q}=3 / 2)$. The meaning of the curves is analogous as described in Fig. 2.

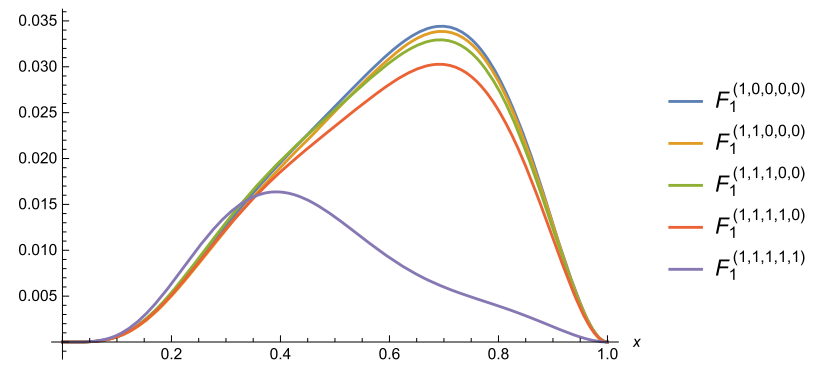

(a) $F_{1}$

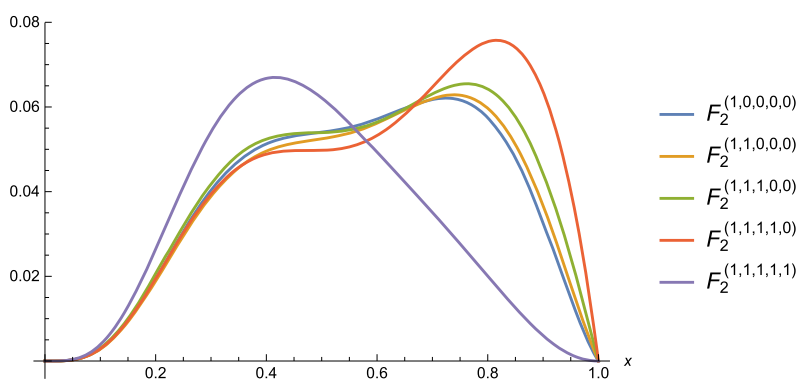

(b) $F_{2}$

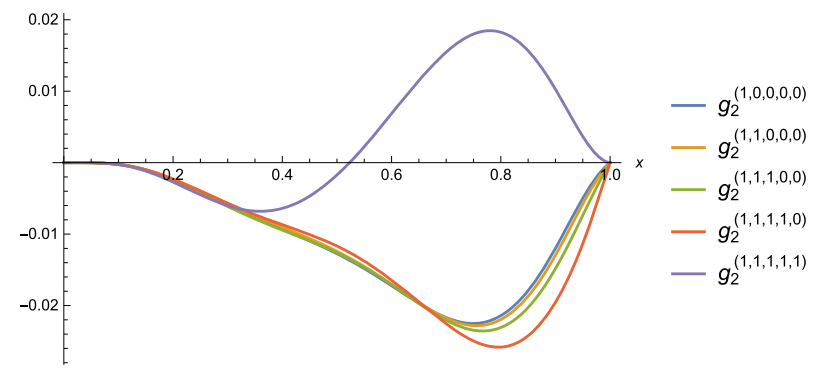

(c) $g_{2}$

FIG. 4. Full structure functions $F_{1}, F_{2}$ and $g_{2}$ as functions of the Bjorken parameter $x$ obtained from the twist-4 spin- $1 / 2$ fermionic operators $\mathcal{O}_{k=1}^{I_{1},(6)}$ with $\left(1, l_{4}, l_{3}, l_{2}, l_{1}\right)_{a=1}$.

associated with the operators $\mathcal{O}_{k=1}^{I_{1},(6)}$ as intermediate hadrons. In contrast, for $0.2 \leq x \leq 0.6$ the leading diagram comes from intermediate states associated with the operators $\mathcal{O}_{k^{\prime}=2}^{I_{2},(13)}$ which belong to the $\mathbf{6 0}$ irreducible representation of $S U(4)_{R}$. The structure functions for states with charge $\mathcal{Q}=3 / 2$ have a different behavior, showing a significant suppression of the contribution of intermediate states associated to $\mathcal{O}_{k=1}^{I_{1},(6)}$ above $x \sim 0.6$ for $F_{1}$ and $F_{2}$. Note that for $g_{1}$ and $g_{2}$ the behavior is different.

\section{B. The case of twist-5 $\mathcal{O}_{k=2}^{I_{2},(6)}=C_{i_{1} i_{2}}^{I_{2},(6)} \operatorname{Tr}\left(F_{+} \lambda_{\mathcal{N}=4} X_{i_{1}} X_{i_{2}}\right)$ operators}

In this subsection we calculate the structure functions for incident hadrons represented by twist $\tau=5$ spin-1/2 fermionic operators $\mathcal{O}_{k=2}^{I_{2},(6)}=C_{i_{1} i_{2}}^{I_{2},(6)} \operatorname{Tr}\left(F_{+} \lambda_{\mathcal{N}=4} X_{i_{1}} X_{i_{2}}\right)$ of the $\mathcal{N}=4$ SYM theory in the planar limit and at strong coupling, for the Bjorken variable within the $\lambda_{\text {SYM }}^{-1 / 2} \ll x<1$ range. The behavior of the different interaction terms in the action (3.7) is very similar to the case $\tau=4$. However, now the number intermediate of hadronic states is much larger. In Fig. 5 we show the structure functions $F_{1}, F_{2}$ and $g_{2}$ for the 15 independent incoming states which can be separated in terms of their charges $\mathcal{Q}=$ $1 / 2,3 / 2,5 / 2$ given by $l_{1}=0,1,2$, respectively. There is only one state $(2,2,2,2,2)_{a}$ with charge $\mathcal{Q}=5 / 2$. Each of these states has associated four spinors, $a=1,2,3,4$. They transform in the $60^{*}$ irreducible representation of $S U(4)_{R}$.

As the value of the charge becomes larger, the Pauli contribution with a maximum in $x \sim 0.7$ decreases. 


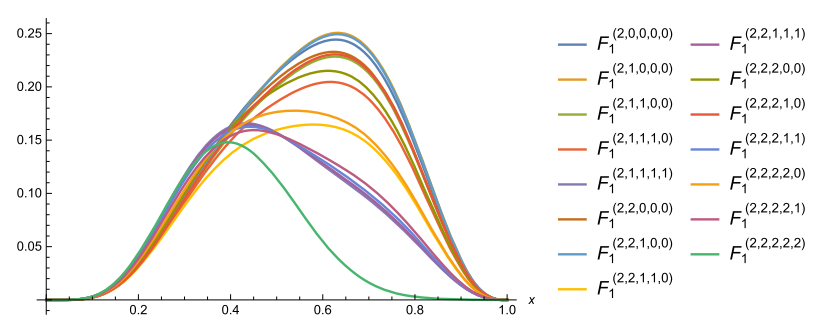

(a) $F_{1}$

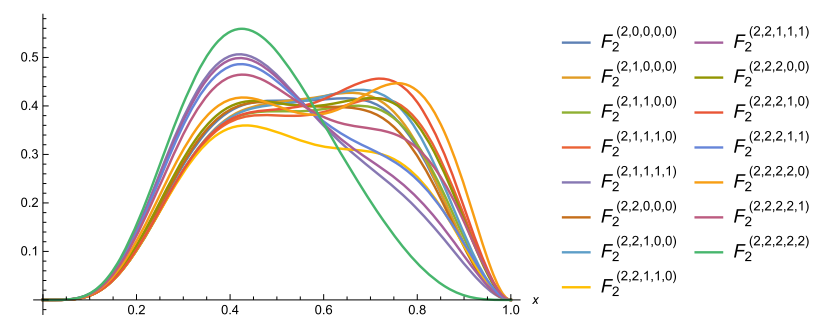

(b) $F_{2}$

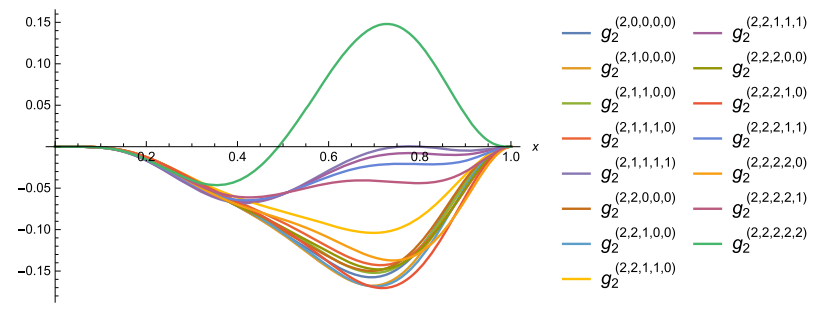

(c) $g_{2}$

FIG. 5. Full structure functions $F_{1}, F_{2}$ and $g_{2}$ as functions of the Bjorken parameter $x$ obtained from the twist-5 spin-1/2 fermionic operators $\mathcal{O}_{k=2}^{I_{2},(6)}$ with $\left(2, l_{4}, l_{3}, l_{2}, l_{1}\right)_{a=1}$.

This effect occurs mainly due to two reasons, which are related to the previous case for $\tau=4$. First, the states which have the biggest $\mathcal{Q}$ couple to a smaller number of intermediate states due to charge conservation. They do not even have interactions for $\mathcal{Q}=5 / 2$. The second reason is related to the charge appearing in the coefficient of the minimal coupling and the cross terms. The last contribution suppresses the Pauli term contributions in $F_{1}$ and $F_{2}$ and changes the curve of the $g_{2}$ function for the state $(2,2,2,2,2)$.

It is interesting to analyze the characteristic scale corresponding to the structure functions associated with operators with different twists. Figures have been normalized using the same prescription proposed in Ref. [10] for the minimal $\tau=3$. In this case we have set

$$
\left|a_{0}\right|^{2}\left(\frac{\Lambda^{2}}{q^{2}}\right)^{\tau-1}=\pi^{2} K^{2} 4^{\tau-1} c_{\chi}^{2} c_{i}^{2}\left(\frac{\Lambda^{2}}{q^{2}}\right)^{\tau-1}=1 .
$$

The idea is to study all the contributions from different couplings corresponding to an operator of a given twist. However, using this normalization the maximum values of the structure functions increase for higher-twist operators due to the factor $(\Gamma(\tau))^{2}$. For instance, the Pauli contribution $F_{2}^{P}$ (see Appendix A) takes the form

$$
\begin{aligned}
F_{2}^{P}(x, q, \tau) \sim & \pi^{2} K^{2} 4^{\tau-1} c_{\chi}^{2} c_{i}^{2}\left(\frac{\Lambda^{2}}{q^{2}}\right)^{\tau-1}(\Gamma(\tau))^{2} \\
& \times x^{\tau+1}(1-x)^{\tau-2}[1+\tau(\tau-2) x]
\end{aligned}
$$

where the factor $(\Gamma(\tau))^{2}$ scales the contributions, and it increases for higher twists, considering Eq. (3.36).
In order to clarify this behavior, let us recall that the normalization of the structure functions depends on $\tau$ and the calculation has been carried out in the DIS limit $\left(q \sim M_{\chi} \gg M_{i} \sim \Lambda_{\mathrm{QCD}}\right)$. The standard normalization used in $[4,47]$ implies that the constant $C$ in Eq. (3.17) is written in terms of the twist $\tau$ and the hadronic mass $M_{\tau, i}$ as follows:

$$
C=\frac{\sqrt{2}}{z_{0} J_{\tau-1}\left(M z_{0}\right)} .
$$

Thus, the normalization constant of the incident hadrons is $c_{i}=\frac{\sqrt{2}\left(M_{i} z_{0}\right)^{\tau-2}}{J_{\tau-1}\left(M_{i} z_{0}\right) 2^{\tau-2} \Gamma(\tau-1)}$, while for the intermediate state, by using the asymptotic expansion of the Bessel function around $M_{\chi} z_{0} \gg 1$, we obtain $c_{\chi} \sim \pi$. Finally, we can calculate the structure function $F_{2}^{P}$ evaluated in the maximum $x_{\max }=\frac{1+\tau}{2 \tau-1}$ with the full $\tau$ dependence of the normalization constants, obtaining

$$
\begin{aligned}
F_{2}^{P}\left(x_{\max }, q, \tau\right) \sim & \frac{K^{2} \tau^{2}}{\left(M_{i} z_{0}\right)^{2}\left(J_{\tau-1}\left(M_{i} z_{0}\right)\right)^{2}}\left(\frac{M_{i}^{2}}{q^{2}}\right)^{\tau-1} \\
& \times x_{\max }^{\tau+1}\left(1-x_{\max }\right)^{\tau-2}\left(1+x_{\max } \tau(\tau-2)\right),
\end{aligned}
$$

where $M_{i}$ is the mass of the incident hadron and it depends on the $j$ th zero of the Bessel function. ${ }^{3}$ The maximum value of the contribution scales with $\left(M_{i}^{2} / q^{2}\right)^{\tau-1}$. Thus, it becomes evident how the falloff is accentuated for

\footnotetext{
${ }^{3}$ For each $\tau$, there is a mass tower of hadrons $M_{\tau, j}$ which is given by the $j$ th zero of the Bessel functions $J_{\tau-2}\left(R_{\tau, j}\right)=0$ (hard-wall model), where $R_{\tau, j}=M_{\tau, j} z_{0}$.
} 
higher-twist operators. For example, phenomenologically we can consider the kinematic variables taking the values $q^{2} \sim 15 \mathrm{GeV}^{2}, M_{i} \sim 1 \mathrm{GeV}$ and $\Lambda_{\mathrm{QCD}} \sim 0.2 \mathrm{GeV}$. Therefore, if we consider the lower hadronic mass for each $\tau$, the maximum value for $\tau=3$ is approximately 8.6 times greater than the case $\tau=4$, and 47 times greater than the case $\tau=5$. These results may be important to compare with the QCD phenomenology [48].

\section{COMMENTS ON THE RESULTS AND CONCLUSIONS}

We have done an exhaustive study of different contributions to the structure functions of electromagnetic DIS off polarized spin-1/2 hadrons using the AdS/CFT duality. Particularly, we have focused on local single-trace highertwist spin-1/2 fermionic operators of the strongly coupled $S U(N) \mathcal{N}=4$ SYM theory in the planar limit. Specifically, we have worked out in full detail the cases with $\tau=4$ and 5. It is worth mentioning that we have carried out all our calculations from first principles, i.e., considering the background $\mathrm{AdS}_{5} \times S^{5}$ from type IIB supergravity. In the effective five-dimensional action (3.7) there are several contributions. The first one comes from the minimalcoupling term. In addition, there are very important contributions from the second and third terms which are Pauli interactions. For all structure functions the contributions obtained from the minimal-coupling term turn out to be smaller in comparison with those emerging from the different Pauli terms. This effect is manifested on both the symmetric as well as the antisymmetric structure functions, as shown in all the figures displayed in this work. In comparison with the case of $\mathcal{O}_{0}^{I_{0},(6)}$ operators in the $4^{*}$ irreducible representation of $S U(4)_{R}$, which are only four different operators, and consequently there are only four different dual Kaluza-Klein dilatino modes, in this work for $\tau=4$, the operators are in the $\mathbf{2 0}^{*}$ irreducible representation of $S U(4)_{R}$ and the dual corresponding fermionic type IIB supergravity modes are also 20. For $\tau=5$ these figures become 60 SYM operators and their corresponding 60 Kaluza-Klein states, respectively. On the other hand, calculations become much more complicated for twists 4 and 5 in comparison with 3 . In addition, there are new relevant terms from the selection rule $\lambda_{k} \rightarrow \lambda_{k-1}$ with $k>0$. All this has been discussed in detail in the previous sections.

Another interesting point to mention concerns the OPE of two electromagnetic currents inside the hadron. Its matrix elements define the tensor (1.4), which can be expanded in terms of the generic structure functions $\tilde{F}_{i}\left(x, q^{2} / \Lambda^{2}\right)$. The optical theorem leads to Eq. (1.6), thus the relation with the usual structure functions defining the hadronic tensor $W_{\mu \nu}$ is $F_{i}\left(x, q^{2} / \Lambda^{2}\right) \equiv 2 \pi \operatorname{Im} \tilde{F}_{i}\left(x, q^{2} / \Lambda^{2}\right)$. Then, we can obtain the moments of the structure functions $M_{n}^{i}\left(q^{2}\right)$, which can be schematically written as the sum of three kinds of contributions [4]. The leading contribution at weak coupling comes from twist-2 operators. In Ref. [49] the DGLAP and BFKL evolution equations in the $\mathcal{N}=4$ SYM theory at the next-to-leading approximation have been derived. Also, the evaluation of Wilson coefficients for DIS has been done in the next-to-leading approximation in [50]. There are other contributions to the moments of the structure functions $M_{n}^{i}\left(q^{2}\right)$ from the nonperturbative domain, which are the ones we have considered in the present work. These contributions come from double-trace operators constructed from protected single-trace operators. In the present case these are the protected single-trace twist$\tau$ (for $\tau \geq 3$ ) spin- $1 / 2$ operators of $\mathcal{N}=4$ SYM theory at strong coupling of the form $\mathcal{O}_{k}^{I_{k},(6)}$ which belong to the $4^{*}, \mathbf{2 0}^{*}, \mathbf{6 0}^{*}, \ldots$ irreducible representations of $S U(4)_{R}$, labeled as $[1, k, 0]$. As we have seen in previous sections there are also contributions from operators of the form $\mathcal{O}_{k}^{I_{k},(13)}$ in the $4, \mathbf{2 0}, \mathbf{6 0}, \ldots$ irreducible representations of $S U(4)_{R}$, labeled as $[0, k, 1]$. These contributions to the OPE are the leading ones in the large- $N$ limit and correspond to final single-particle states in DIS or the exchange of singleparticle intermediate states in the forward Compton scattering. In terms of the type IIB supergravity dual description these processes only involve a single intermediate dilatino mode exchange. This is what we have developed in this work.

There is a third kind of contributions which are relevant for finite $N$, and they correspond to the exchange of two or more particle intermediate states in the forward Compton scattering. They correspond to multitrace operators in the SYM theory. In the present case we have not considered $1 / N$ corrections since here we focus on the large- $N$ limit of the $S U(N) \mathcal{N}=4$ SYM theory. We have obtained the structure functions for exchange of two-particle intermediate states for glueballs in [21] for $\mathcal{N}=4$ SYM theory in terms of type IIB supergravity on $\mathrm{AdS}_{5} \times S^{5}$, scalar mesons in [22] and vector mesons in [23] both in the context of the D3D7-brane model [11].

An important point is related to the constituent counting rules. For instance, in Ref. [51] it has been proposed a phenomenological light-front quark model consistent with the Drell-Yan-West duality [52,53] and quark counting rules $[54,55]$. They have obtained the correct scaling behavior of parton distributions and form factors for pions and nucleons. Moreover in [56], using global fits for valence light-quark parton distributions and data on quark and nucleon form factors in the Euclidean region, a lightfront quark model for the nucleon structure has been developed. More recently in Ref. [57], using light-front holographic QCD, generalized parton distributions have been investigated. It has been included a certain reparametrization function to account for Regge behavior at small $x$ and inclusive counting rules at $x$ near 1 . 
At this point we should emphasize that a property derived from the type IIB supergravity dual description which we consider in the present work is the fact that for fermions the structure function $F_{2}$ scales as $(1-x)^{\tau-2}$ for $x \rightarrow 1$. In that parametric regime this is related to the fact that the OPE of two electromagnetic currents inside a baryon is dominated by double-trace operators of $\mathcal{N}=4$ SYM theory in the planar limit. It is understood as if the charged lepton is scattered from a single entire baryon rather than its partonic constituents [4]. Recall that this top-down approach holds within the range $\lambda_{\text {SYM }}^{-1 / 2} \ll x<1$, where the 't Hooft coupling satisfies the condition $N \gg \lambda_{\text {SYM }} \gg 1$. A similar behavior takes place for glueballs for the structure function $F_{2}$ as $x \rightarrow 1$. On the other hand, in the case of the low-lying holographic dual mesons the structure function $F_{2}$ scales as $(1-x)^{\gamma}$ for $x \rightarrow 1$, being the exponent $\gamma$ dependent on the particular flavor Dp-brane model one considers. In Ref. [20] it has been obtained $F_{2} \propto(1-x)^{2}$ in that limit for the D3D7-brane model, which is consistent with the constituent counting rules for the pion commented before. For the Sakai-Sugimoto model based on D4D8 anti-D8-brane system the exponent is $\gamma \approx 4.59$, while the D4D6 anti-D6-brane model leads to $\gamma \approx 3.33$. Thus, it allows to distinguish the D3D7-brane model as the one which reproduces correctly the constituent counting rule for the pion. The reason for that is that even in the large- $N$ limit mesons have only a quark-antiquark pair, and in the dual holographic representation there is a fundamental string connecting them. On the other hand, fundamental strings connecting $N$ quarks to a baryon vertex lead to a nondynamical baryon in the large- $N$ limit, whose mass is proportional to $N$. Thus, in the range $\lambda_{\text {SYM }}^{-1 / 2} \ll x<1$ a holographic dynamical baryon is usually represented in terms of a totally different construction, given by the dilatino of type IIB supergravity. The dilatino is decomposed in terms of its Kaluza-Klein modes upon the compactification on $S^{5}$. Beyond this parametric domain, for instance when $\exp \left(-\lambda_{\text {SYM }}^{1 / 2}\right) \ll x \ll \lambda_{\text {SYM }}^{-1 / 2}$, the holographic description is given by an ultralocal approximation of the type IIB superstring theory four-point scattering amplitude of two graviphotons and two fermions [9]. In addition, for exponentially small values of the Bjorken parameter the holographic description is carried out in terms of the Brower-Polchinski-Strassler-Tan (BPST) Pomeron [58].

Another important point concerns the study of the Callan-Gross relation $F_{2}=2 x F_{1}$ within this holographic dual approach. Figure 6 displays $F_{2} /\left(2 x F_{1}\right)$ as a function of $x$ by considering different twists: $\tau=3$ indicated with a blue line; $\tau=4$ for an incoming state $(1,1,1,1,1)$ (orange curve); and for $\tau=5$ setting $(2,2,2,2,2)$ as the incoming state (green line). For $\tau=3$ there is a clear trend towards the Callan-Gross relation as the Bjorken variable increases. In the case with $\tau=4$ there are some deviations due to

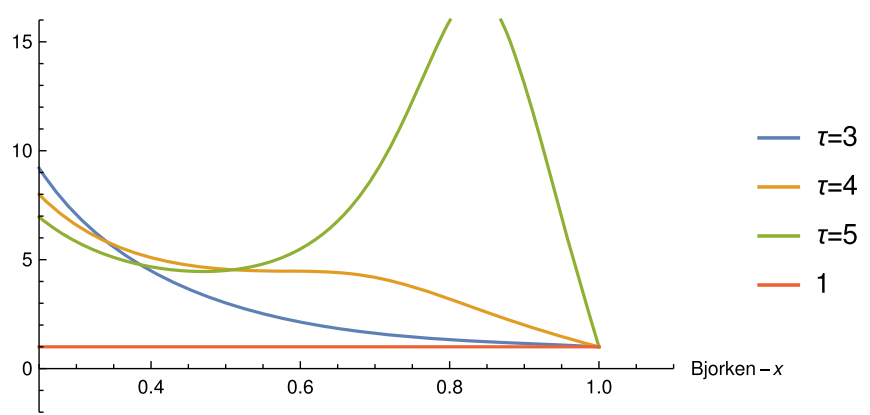

FIG. 6. $\quad F_{2} /\left(2 x F_{1}\right)$ as a function of the Bjorken parameter $x$ for different values of the operator twist. The horizontal red line indicates the Callan-Gross relation, i.e., $F_{2} /\left(2 x F_{1}\right)=1$.

additional contributions coming from terms which mix the incoming and intermediate states in the forward Compton scattering, however we still can observe a general trend as $x \rightarrow 1$. The odd behavior for $\tau=5$ is due to the term resulting from the supergravity Feynman diagram for the forward Compton scattering which mixes one vertex bearing the minimal coupling and the other one with the Pauli interaction. The general increase shown for small values of $x$ should not be considered as the correct dual description, since the supergravity approach should be replaced by the string theory/BPST Pomeron description in the range $0<x<0.1$, where the upper limit is a typical value. It is interesting to recall that if we only turn on the minimal interaction the Callan-Gross relation becomes $F_{2}=2 F_{1}$, i.e., $x$ is not present [4].

Now, we can carry out a comparison with experimental data for the proton structure function $F_{2}$. Blue dots correspond to data from the SLAC collaboration [59] for $x=0.85$. Yellow and light-blue dots for $q^{2}<30 \mathrm{GeV}^{2}$ also correspond to the same experiment. The rest of the points belong to the BCDMS collaboration [60].

It is interesting to also comment about previous results [61] where unpolarized spin- $1 / 2$ baryonic structure functions have been investigated in the regime of large Bjorken parameter $x$, by using an exponential deformation of the $\mathrm{AdS}_{5}$ spacetime within the bottom-up AdS/QCD approach. This is related to an anomalous dimension introduced in that model. For the values of $x=0.65,0.75$ and 0.85 their results are consistent with experimental data $[59,60]$ as shown in Fig. 5 of Ref. [61]. This model includes a set of three parameters for each value of $x$, therefore, they have a total of nine parameters to fit the experimental data mentioned above.

In order to make some comparison with the results of Ref. [61] we draw Fig. 7, which displays the experimental data of the structure function $F_{2}$ for the proton, including the corresponding error bars. Colors correspond to different values of the Bjorken parameter for the same three values considered in that paper: $x=0.65,0.75$ and 0.85 . The horizontal axis labels the logarithm of $q^{2}$. The long-dashed 


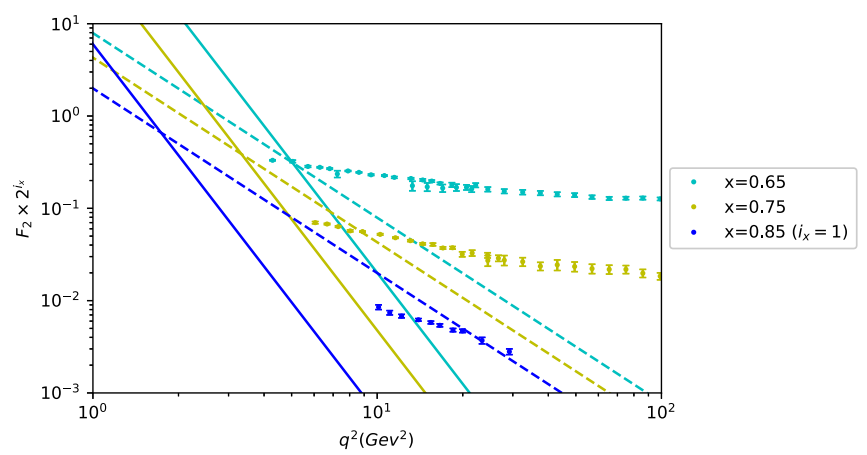

FIG. 7. Data of the structure function $F_{2}$ for the proton extracted from Refs. [59,60], as a function of $\log q^{2}$. Longdashed lines represent the best fit for $\tau=3$. Continuous lines correspond to the best fit for twist-5 operator corresponding to the Kaluza-Klein state $(2,2,2,2,2)$. Dotted lines (very close to the long-dashed lines) correspond to a fit with a linear combination of twist 3, 4 and 5 operators as explained in the main text. Note that in this figure $F_{2}$ has been multiplied by $2^{i_{x}}$, where $i_{x}$ is the number of the $x$ bin, ranging from $1(x=0.85)$ to $3(x=0.65)$.

lines correspond to the best fit for $\tau=3$, obtaining the overall normalization constant $A_{\tau=3}=160 \pm 12$. On the other hand, the continuous lines correspond to the best fit for $\tau=5$, where for the overall normalization constant we obtain $A_{\tau=5}=179 \pm 43$. This fit certainly is not good, as expected if one tries to describe in this parametric regime the proton structure function $F_{2}$ in terms of twist-5 operators, omitting the leading contribution from twist-3 operators. The case for twist- 4 operators lies in between, and we do not display the corresponding curves to make Fig. 7 visually clearer.

At this point we may ask how the combined contributions of twist 3, 4 and 5 single-trace operators of $\mathcal{N}=4$ SYM theory can describe the proton structure function $F_{2}$, by considering the following expression:

$$
\begin{aligned}
F_{2}\left(x, q^{2}\right)= & A_{\tau=3} F_{2}^{\tau=3}\left(x, q^{2}\right)+A_{\tau=4} F_{2}^{\tau=4}\left(x, q^{2}\right) \\
& +A_{\tau=5} F_{2}^{\tau=5}\left(x, q^{2}\right),
\end{aligned}
$$

where for $\tau=4$ we have considered the Kaluza-Klein supergravity state labeled as $(1,1,1,1,1)$, while for $\tau=5$ we set $(2,2,2,2,2)$. The best fit parameters we obtain are

$$
\begin{aligned}
& A_{\tau=3}=156 \pm 38, \quad A_{\tau=4}=10 \pm 237, \\
& A_{\tau=5}=0 \pm 136 .
\end{aligned}
$$

Notice that the fit has been restricted to positive values of the coefficients $A_{\tau}$. The results are shown using dotted lines which are very close to the long-dashed lines corresponding to the twist 3. The large errors for $A_{\tau=4}$ and $A_{\tau=5}$ indicate that the combined fit worsens in comparison to the case considering only the leading-twist operator $\tau=3$. This suggests that this particular top-down holographic dual model is not able to capture the behavior of the proton structure function $F_{2}$ for large values of the Bjorken parameter, and that this situation does not improve by considering the next subleading higher-twist contributions, at least in the planar limit. Let us emphasize that we are comparing a holographic dual model of a confining IR deformation of $\mathcal{N}=4$ SYM theory, which is not QCD, and moreover their differences (at least at the level of their structure functions) become more evident within the parametric domain we consider in this work. It may be possible that nonplanar contributions, whose holographic dual description corresponds to one-loop Feynman diagrams from the supergravity side, could improve the situation, as it occurs for the pion [22] and the rho meson [23]. However, a first principles calculation of this kind for spin- $1 / 2$ fermions is far beyond the results presented in this work. In addition, when comparing Fig. 7 with the results of Ref. [61] we should keep in mind that the AdS/QCD model of that paper uses nine free parameters to fit data from $[59,60]$. On the other hand, the top-down type IIB supergravity dual description we consider has only one free parameter for each twist. In the combined situation there are only three free parameters $A_{\tau=3}, A_{\tau=4}, A_{\tau=5}$, while for our long-dashed curves there is only one free parameter $A_{\tau=3}$ related to the fit of all data. The reason for having only a few parameters is inherent to the nature of the top-down holographic dual description, ultimately derived from superstring theory.

It is very interesting to make some comparison with previous calculations carried out with soft-wall approaches as in Refs. [62,63]. First let us recall that we have explicitly obtained all the structure functions, considering all possible contributions from the hard-wall model description based on type IIB supergravity on $\operatorname{AdS}_{5} \times S^{5}$, by matching the corresponding Kaluza-Klein fermionic modes from the dilatino to certain specific single-trace operators of $\mathcal{N}=4$ SYM theory. Thus, as explained before, the OPE of two electromagnetic currents inside the hadron is dominated by terms with double-trace operators. These doubletrace operators are constructed from the above-mentioned protected single-trace operator with a given twist, and we explicitly calculate their contributions to all the structure functions. Finally, we have associated the obtained structure functions with the ones of the proton, for large $x$ and strong 't Hooft coupling within the planar limit. The relation we have considered between the structure functions which we have derived from single-trace operators of $\mathcal{N}=4$ SYM theory and those of the proton has a number of important limitations, coming from the fact that $\mathcal{N}=4$ SYM theory is not QCD. Still, it is very interesting to make a comparison between them as we have done.

Now, let us comment on other calculations previously done in terms of soft-wall approaches. In Ref. [62] it has been proposed an identification of the higher spin hadrons with the fluctuations around the spin $0,1 / 2,1$ and $3 / 2$ 
string solutions on $\mathrm{AdS}_{5} \times S^{5}$. They have obtained the hadronic spectrum. For that, they identify hadrons by the correspondence of string modes with the dimension of the interpolating operator of the hadrons valence Fock state. Another very interesting investigation of nucleon electromagnetic and axial form factors which has been carried out using the soft-wall model is presented in [63]. In that paper, by setting to 3 the number of colors the nucleon structure is described in a superposition of a 3 valence quark state with high Fock states including an adjustable number of partons. This has been done by considering five-dimensional fermions bearing different scaling dimension in $\mathrm{AdS}_{5}$. The scaling dimension is associated with the Fock state components with a specific number of partons, and particularly they considered the contribution of three, four and five parton components in the nucleon Fock state. They found a reasonable level of agreement in comparison with data for the nucleon form factors. The ideas of Refs. [62,63] are conceptually different from what we have developed in the present work.

The techniques presented in this work can be used to study other single-trace operators of the $S U(N) \mathcal{N}=4$ SYM theory. Also, it would be very interesting to extend it to study cases with less supersymmetries such as $\mathcal{N}=1$ SYM theory developed by Klebanov and Witten [64], considering the spectrum of type IIB supergravity on $\mathrm{AdS}_{5} \times T^{1,1}[65,66]$. In this case the angular integrals on $T^{1,1}$ would set different selection rules which likely induce new interesting effects. In similar lines it would be interesting to investigate the case of type I' string theory associated with five-dimensional supersymmetric fixed points with $E_{N_{f+1}}$ global symmetry. This includes many different theories with $E_{N_{f+1}}=E_{8}, E_{7}, E_{6}, E_{5}=\operatorname{Spin}(10)$,
$E_{4}=S U(5), E_{3}=S U(3) \times S U(2), E_{2}=S U(2) \times U(1)$, $E_{1}=S U(2)$ global symmetry groups [67]. The gravity duals of these theories were constructed in [68], and are related to the near horizon limit of the D4D8-brane system in massive type IIA supergravity [69] compactified on the $\mathrm{AdS}_{6} \otimes S^{4}$ fibration [70]. Interesting related gauge/ supergravity duals have been obtained in [71]. Also, using the ideas discussed above, it would be interesting to investigate eleven-dimensional supergravity on $\mathrm{AdS}_{4} \times S^{7}$ and $\mathrm{AdS}_{7} \times S^{4}$ [72-74] as well as deformations leading to SYM theories preserving less supersymmetries [75].

\section{ACKNOWLEDGMENTS}

We thank Gustavo Michalski for collaboration in the early stages of the project and for a critical reading of the manuscript. This work has been supported in part by the National Scientific Research Council of Argentina (CONICET), the National Agency for the Promotion of Science and Technology of Argentina (ANPCyT-FONCyT) Grants No. PICT-2015-1525 and No. PICT-2017-1647, the UNLP Grant No. PID-X791, and the CONICET Grants PIP-UE Búsqueda de nueva física and PICT-E 20180300 (BCIE).

\section{APPENDIX A: MINIMAL COUPLING AND PAULI TERMS CONTRIBUTIONS TO THE STRUCTURE FUNCTIONS}

In this Appendix we introduce the structure functions associated with each interaction term which are used to draw the figures for higher-twist spin- $1 / 2$ operators. For the minimal coupling the structure functions of a incident polarized spin-1/2 hadron with twist $\tau$ are given by

$$
\begin{gathered}
F_{1}^{m}=\frac{F_{2}^{m}}{2}=\frac{F_{3}^{m}}{2}=g_{1}^{m}=\frac{g_{3}^{m}}{2}=\frac{g_{4}^{m}}{2}=\frac{g_{5}^{m}}{2}=\frac{\left|a_{0}\right|^{2}}{8} \Gamma^{2}(\tau)\left(\frac{\Lambda^{2}}{q^{2}}\right)^{\tau-1} x^{\tau+1}(1-x)^{\tau-2}, \\
g_{2}^{m}=\left(\frac{1}{2} \frac{\tau+1}{\tau-1}-\frac{x \tau}{\tau-1}\right) \frac{\left|a_{0}\right|^{2}}{8} \Gamma^{2}(\tau)\left(\frac{\Lambda^{2}}{q^{2}}\right)^{\tau-1} x^{\tau}(1-x)^{\tau-2},
\end{gathered}
$$

where $\Gamma(x)$ is the Gamma function and $\left|a_{0}\right|=2 \pi c_{i}^{\prime} c_{\mathcal{X}}^{\prime} 2^{\tau} K$ is a constant.

For the Pauli interactions between states in the same representation the structure functions are

$$
\begin{gathered}
F_{1}^{P}=\frac{F_{3}^{P}}{2}=g_{1}^{P}=\frac{g_{5}^{P}}{2}=\frac{1}{2}\left|a_{0}\right|^{2} \Gamma^{2}(\tau)\left(\frac{\Lambda^{2}}{q^{2}}\right)^{\tau-1} x^{\tau+1}(1-x)^{\tau-2}(1-\tau)^{2}, \\
F_{2}^{P}=g_{4}^{p}=\left|a_{0}\right|^{2} \Gamma^{2}(\tau)\left(\frac{\Lambda^{2}}{q^{2}}\right)^{\tau-1} x^{\tau+1}(1-x)^{\tau-2}(1+x \tau(\tau-2)), \\
g_{2}^{P}=-\frac{1}{4}\left|a_{0}\right|^{2} \Gamma^{2}(\tau)\left(\frac{\Lambda^{2}}{q^{2}}\right)^{\tau-1}(1-x)^{\tau-2} x^{\tau+1}(\tau(1-\tau+x(3+2(\tau-2) \tau))-1) /(\tau-1),
\end{gathered}
$$




$$
g_{3}^{P}=-\left|a_{0}\right|^{2} \Gamma^{2}(\tau)\left(\frac{\Lambda^{2}}{q^{2}}\right)^{\tau-1}(1-x)^{\tau-2} x^{\tau+1}\left(1+\tau(1-3 x)+(2 x-1) \tau^{2}\right) /(\tau-1) .
$$

Finally, the structure functions from the cross-terms contribution having both the minimal coupling and the Pauli interactions are

$$
\begin{gathered}
F_{1}^{c}=\frac{F_{3}^{c}}{2}=g_{1}^{c}=\frac{g_{5}^{c}}{2}=\frac{1}{2}\left|a_{0}\right|^{2}\left(\frac{\Lambda^{2}}{q^{2}}\right)^{\tau-1}(1-x)^{\tau-2} x^{\tau+1}(\tau-1) \Gamma(\tau)^{2}, \\
F_{2}^{c}=g_{4}^{c}=\left|a_{0}\right|^{2}\left(\frac{\Lambda^{2}}{q^{2}}\right)^{\tau-1}(1-x)^{\tau-2} x^{\tau+1}(-1+x \tau) \Gamma(\tau)^{2}, \\
g_{2}^{c}=-\frac{1}{4}\left|a_{0}\right|^{2}\left(\frac{\Lambda^{2}}{q^{2}}\right)^{\tau-1} \frac{\Gamma(\tau)^{2}}{\tau-1}(1-x)^{\tau-2} x^{\tau+2}\left(2-\tau^{2}+x \tau(4 \tau-5)\right), \\
g_{3}^{c}=\frac{1}{2} \frac{\Gamma(\tau)^{2}}{\tau-1}\left|a_{0}\right|^{2}\left(\frac{\Lambda^{2}}{q^{2}}\right)^{\tau-1}(1-x)^{\tau-2} x^{\tau+1}\left(2-(4+x) \tau+(-1+4 x) \tau^{4}\right) .
\end{gathered}
$$

\section{APPENDIX B: TABLES OF ANGULAR INTEGRALS}

The coefficients corresponding to the terms in the action (3.7) are calculated from the angular integrals of the spinor spherical harmonics and the Killing vectors $v^{\alpha}$. The results of the following integrals are shown in Table I,

$$
\int d \Omega_{5}\left(\Theta_{\left(1, l_{4}^{\prime}, l_{3}^{\prime}, l_{2}^{\prime}, l_{1}^{\prime}\right)_{a}}^{-}\right)^{*} v^{i} \tau_{i} \Theta_{\left(1, l_{4}, l_{3}, l_{2}, l_{1}\right)_{a=1}}^{-}
$$

where the incoming spinor spherical harmonics is $\Theta_{\left(1, l_{4}, l_{3}, l_{2}, l_{1}\right)_{a=1}}^{-}$while the outgoing one is given by $\Theta_{\left(1, l_{4}^{\prime}, l_{3}^{\prime}, l_{2}^{\prime}, l_{1}^{\prime}\right)_{a}}^{-}$. In this case both of them belong to the same Kaluza-Klein mass tower.

Then, in Tables II and III are listed the results of the following integrals between states belonging to different KaluzaKlein mass towers:

$$
\int d \Omega_{5}\left(\Theta_{\left(1 \pm 1, l_{4}^{\prime}, l_{3}^{\prime}, l_{2}^{\prime}, l_{1}^{\prime}\right)_{a}}^{+}\right)^{*} v^{i} \tau_{i} \Theta_{\left(1, l_{4}, l_{3}, l_{2}, l_{1}\right)_{a=1}}^{-}
$$

In this case the outgoing spinors have superscript $(+)$ and $l_{5}$ can only take the values $1 \pm 1$.

TABLE I. Results of the angular integrals between states belonging to the same Kaluza-Klein mass tower. The spinor spherical harmonics corresponding to the incoming states are indicated in the first row. The outgoing states are listed in the first column.

\begin{tabular}{lccccc}
\hline \hline & $(1,0,0,0,0)_{1}^{-}$ & $(1,1,0,0,0)_{1}^{-}$ & $(1,1,1,0,0)_{1}^{-}$ & $(1,1,1,1,0)_{1}^{-}$ & $(1,1,1,1,1)_{1}^{-}$ \\
\hline$(1,0,0,0,0)_{1}^{-}$ & $-\frac{7}{30}$ & $-\frac{1}{20 \sqrt{6}}$ & 0 & 0 \\
$(1,0,0,0,0)_{3}^{-}$ & 0 & 0 & $\frac{1}{12 \sqrt{10}}$ & $\frac{1}{12 \sqrt{5}}$ & 0 \\
$(1,1,0,0,0)_{1}^{-}$ & $-\frac{1}{20 \sqrt{6}}$ & $-(9 / 40)$ & 0 & $\frac{1}{4 \sqrt{30}}$ & 0 \\
$(1,1,0,0,0)_{3}^{-}$ & 0 & 0 & $\frac{1}{8 \sqrt{15}}$ & $\frac{1}{12 \sqrt{2}}$ & 0 \\
$(1,1,1,0,0)_{1}^{-}$ & 0 & 0 & $-\frac{5}{24}$ & $-\frac{1}{6}$ & 0 \\
$(1,1,1,0,0)_{3}^{-}$ & $-\frac{1}{12 \sqrt{10}}$ & $-\frac{1}{8 \sqrt{15}}$ & 0 & 0 \\
$(1,1,1,1,0)_{1}^{-}$ & 0 & 0 & $\frac{1}{12 \sqrt{2}}$ & 0 & 0 \\
$(1,1,1,1,0)_{3}^{-}$ & $\frac{1}{12 \sqrt{5}}$ & 0 & $-\frac{1}{4 \sqrt{30}}$ & 0 & 0 \\
$(1,1,1,1,1)_{1}^{-}$ & 0 & 0 & 0 & 0 \\
$(1,1,1,1,1)_{3}^{-}$ & 0 & 0 & 0 & 0 \\
\hline \hline
\end{tabular}


TABLE II. Results of the angular integrals between states belonging to different Kaluza-Klein mass towers. In the first row there are the incoming spinor spherical harmonics while the outgoing states are listed in the first column.

\begin{tabular}{|c|c|c|c|c|c|}
\hline & $(1,0,0,0,0)_{1}^{-}$ & $(1,1,0,0,0)_{1}^{-}$ & $(1,1,1,0,0)_{1}^{-}$ & $(1,1,1,1,0)_{1}^{-}$ & $(1,1,1,1,1)_{1}^{-}$ \\
\hline$(0,0,0,0,0)_{1}^{+}$ & $-\frac{1}{3 \sqrt{5}}$ & $\frac{1}{\sqrt{30}}$ & 0 & 0 & 0 \\
\hline$(0,0,0,0,0)_{3}^{+}$ & 0 & 0 & $-\frac{1}{3 \sqrt{2}}$ & $-\frac{1}{3}$ & 0 \\
\hline$(2,0,0,0,0)_{1}^{+}$ & $\frac{\sqrt{3}}{10}$ & $-\frac{1}{20 \sqrt{2}}$ & 0 & 0 & 0 \\
\hline$(2,0,0,0,0)_{3}^{+}$ & 0 & 0 & $\frac{1}{4 \sqrt{30}}$ & $\frac{1}{4 \sqrt{15}}$ & 0 \\
\hline$(2,1,0,0,0)_{1}^{+}$ & $\frac{\sqrt{21}}{\sqrt{2} 20}$ & $\frac{9}{40 \sqrt{7}}$ & $\begin{array}{c}4 \sqrt{3} 0 \\
0\end{array}$ & $\begin{array}{c}4 \sqrt{15} \\
0\end{array}$ & 0 \\
\hline$(2,1,0,0,0)_{3}^{+}$ & 0 & 0 & $-\frac{1}{8 \sqrt{105}}$ & $-\frac{1}{4 \sqrt{210}}$ & 0 \\
\hline$(2,1,1,0,0)_{1}^{+}$ & 0 & 0 & $\frac{5}{24 \sqrt{7}}$ & $-\frac{1}{12 \sqrt{14}}$ & 0 \\
\hline$(2,1,1,0,0)_{3}^{+}$ & $\frac{\sqrt{7}}{4 \sqrt{10}}$ & $-\frac{1}{8 \sqrt{105}}$ & 0 & 0 & 0 \\
\hline$(2,1,1,1,0)_{1}^{+}$ & 0 & 0 & $-\frac{1}{12 \sqrt{14}}$ & $\frac{1}{6 \sqrt{7}}$ & 0 \\
\hline$(2,1,1,1,0)_{3}^{+}$ & $-\frac{\sqrt{7}}{4 \sqrt{5}}$ & $\frac{1}{4 \sqrt{210}}$ & 0 & 0 & 0 \\
\hline$(2,1,1,1,1)_{1}^{+}$ & 0 & 0 & 0 & 0 & $\frac{1}{2 \sqrt{7}}$ \\
\hline$(2,1,1,1,1)_{3}^{+}$ & 0 & 0 & 0 & 0 & $\begin{array}{c}2 \sqrt{2} \\
0\end{array}$ \\
\hline$(2,2,0,0,0)_{1}^{+}$ & 0 & $-\frac{\sqrt{5}}{4 \sqrt{7}}$ & 0 & 0 & 0 \\
\hline$(2,2,0,0,0)_{3}^{+}$ & 0 & 0 & $\frac{1}{4 \sqrt{21}}$ & $\frac{1}{2 \sqrt{42}}$ & 0 \\
\hline$(2,2,1,0,0)_{1}^{+}$ & 0 & 0 & $\frac{5}{12 \sqrt{21}}$ & $-\frac{1}{6 \sqrt{42}}$ & 0 \\
\hline$(2,2,1,0,0)_{3}^{+}$ & 0 & $\frac{\sqrt{5}}{4 \sqrt{7}}$ & 0 & 0 & 0 \\
\hline$(2,2,1,1,0)_{1}^{+}$ & 0 & 0 & $\frac{1}{6 \sqrt{42}}$ & $-\frac{1}{3 \sqrt{21}}$ & 0 \\
\hline$(2,2,1,1,0)_{3}^{+}$ & 0 & $\frac{\sqrt{5}}{2 \sqrt{14}}$ & 0 & 0 & 0 \\
\hline$(2,2,1,1,1)_{1}^{+}$ & 0 & 0 & 0 & 0 & $\frac{1}{\sqrt{21}}$ \\
\hline$(2,2,1,1,1)_{3}^{+}$ & 0 & 0 & 0 & 0 & 0 \\
\hline$(2,2,2,0,0)_{1}^{+}$ & 0 & 0 & 0 & 0 & 0 \\
\hline$(2,2,2,0,0)_{3}^{+}$ & 0 & 0 & $\frac{\sqrt{2}}{3 \sqrt{3}}$ & $\frac{1}{6 \sqrt{3}}$ & 0 \\
\hline
\end{tabular}

TABLE III. Results of the angular integrals between states belonging to different Kaluza-Klein mass towers. In the first row there are the incoming spinor spherical harmonics while the outgoing states are listed in the first column.

\begin{tabular}{cccccc}
\hline \hline & $(1,0,0,0,0)_{1}^{-}$ & $(1,1,0,0,0)_{1}^{-}$ & $(1,1,1,0,0)_{1}^{-}$ & $(1,1,1,1,0)_{1}^{-}$ & $(1,1,1,1,1)_{1}^{-}$ \\
\hline$(2,2,2,1,0)_{1}^{+}$ & 0 & 0 & 0 & 0 & 0 \\
$(2,2,2,1,0)_{3}^{+}$ & 0 & 0 & $-\frac{\sqrt{5}}{3 \sqrt{6}}$ & $\frac{1}{3 \sqrt{15}}$ & 0 \\
$(2,2,2,1,1)_{1}^{+}$ & 0 & 0 & 0 & 0 & 0 \\
$(2,2,2,1,1)_{3}^{+}$ & 0 & 0 & 0 & 0 & $\frac{1}{\sqrt{15}}$ \\
$(2,2,2,2,0)_{1}^{+}$ & 0 & 0 & 0 & $\frac{\sqrt{3}}{2 \sqrt{5}}$ & 0 \\
$(2,2,2,2,0)_{3}^{+}$ & 0 & 0 & 0 & 0 & 0 \\
$(2,2,2,2,1)_{1}^{+}$ & 0 & 0 & 0 & 0 & $-\frac{1}{\sqrt{10}}$ \\
$(2,2,2,2,1)_{3}^{+}$ & 0 & 0 & 0 & 0 & 0 \\
$(2,2,2,2,2)_{1}^{+}$ & 0 & 0 & 0 & 0 & 0 \\
$(2,2,2,2,2)_{3}^{+}$ & 0 & 0 & &
\end{tabular}




\section{APPENDIX C: RESULTS OF THE STRUCTURE FUNCTION $g_{3}$}

In this Appendix we show the structure function $g_{3}$ for $\tau=4$ (Figs. 8 and 9) and $\tau=5$ (Fig. 9).

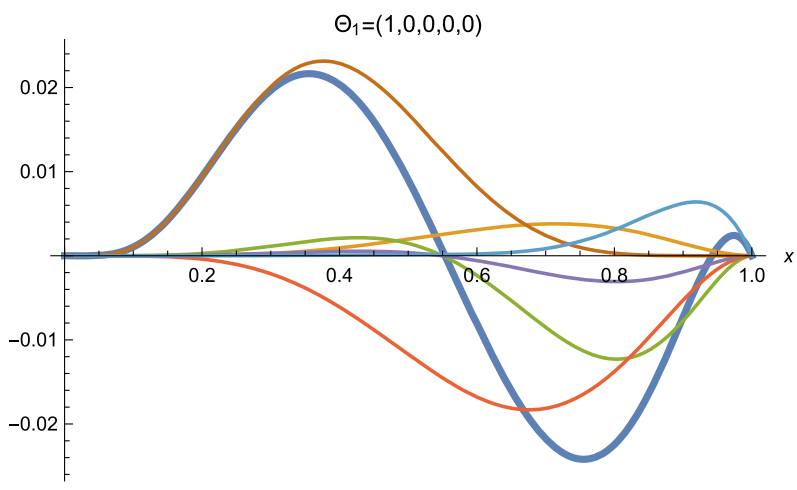

(a) $g_{3}$

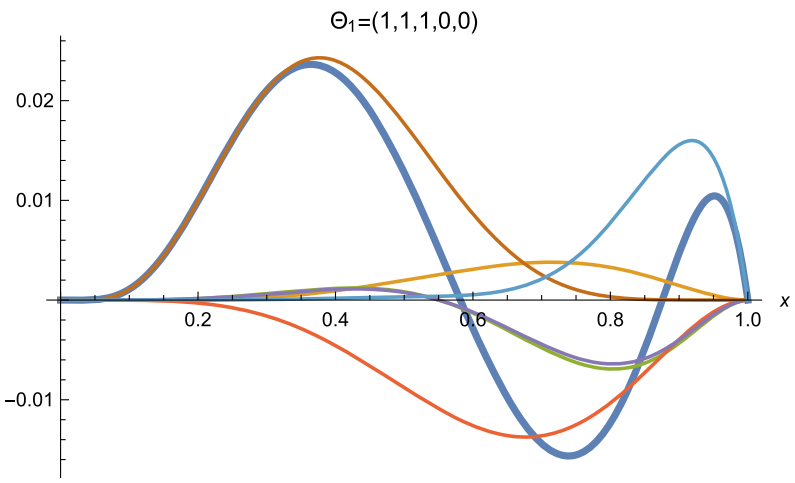

(c) $g_{3}$

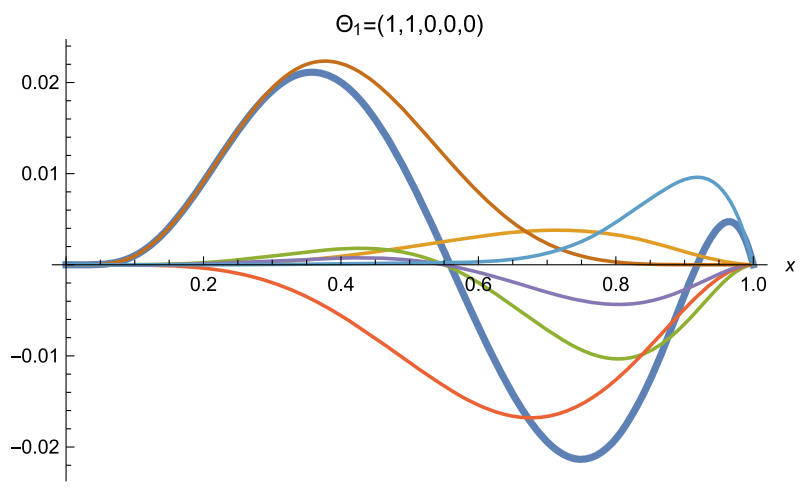

(b) $g_{3}$

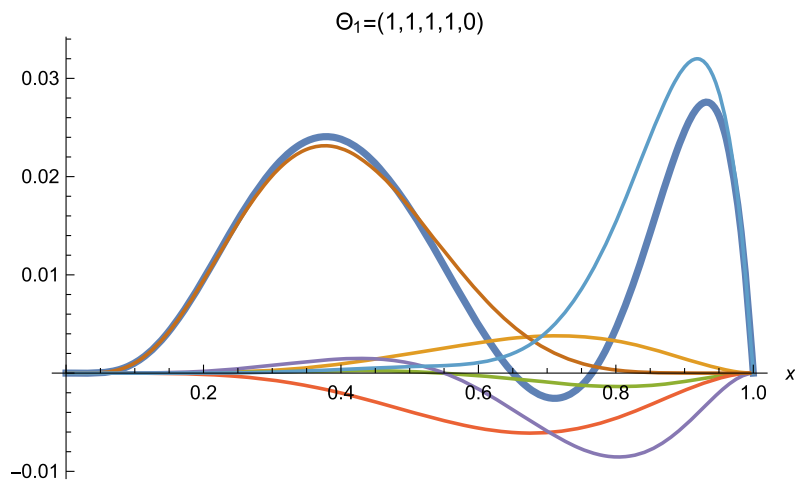

(d) $g_{3}$

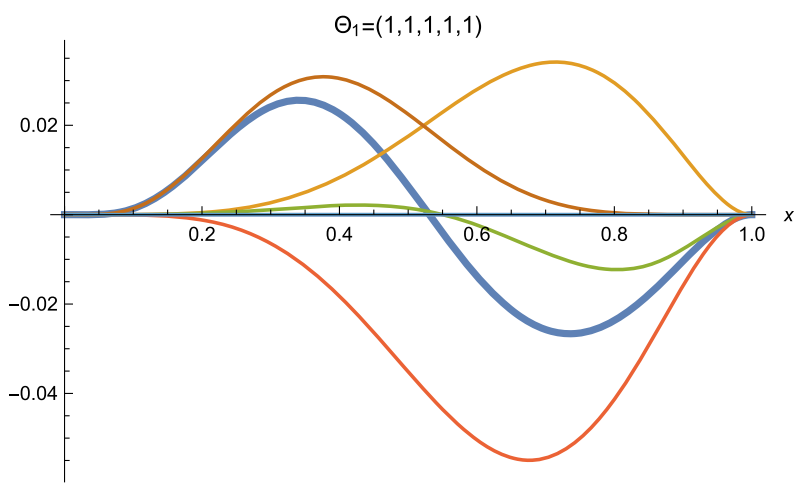

(e) $g_{3}$

FIG. 8. Full structure function $g_{3}$ (blue line) as a function of the Bjorken parameter $x$, obtained from the contribution of the twist-4 spin-1/2 fermionic operators $\mathcal{O}_{k=1}^{I_{1},(6)}$ with $l_{5}=1 \geq l_{4} \geq l_{3} \geq l_{2} \geq l_{1}$. We distinguish the contributions from the minimal coupling $\beta_{m}^{2} F_{1}^{m}$ (orange line); the Pauli interaction between states which belong to the same Kaluza-Klein tower: where the intermediate state $\lambda_{\mathcal{X}} \equiv \lambda_{k=1}^{-}$ is the same as the incident state $\beta_{P}^{2} F_{1}^{P}$ (green line) and where the intermediate state is different $\beta_{P m}^{2} F_{1}^{P}$ (violet line); the contribution from crossed terms $\beta_{m} \beta_{P} F_{1}^{c}$ (red line); and the contributions from the Pauli interaction involving states which are dual to operators belonging to the $\mathbf{6 0}$ irreducible representation of $S U(4)_{R}: \beta_{+}^{2} F_{1}^{P+}$, where $\lambda_{\mathcal{X}} \equiv \lambda_{k+1}^{+}$(brown line), and also the $\mathbf{4}$ irreducible representation of $S U(4)_{R}: \beta_{-}^{2} F_{1}^{P-}$, where $\lambda_{\mathcal{X}} \equiv \lambda_{k-1}^{+}$(light blue line). 


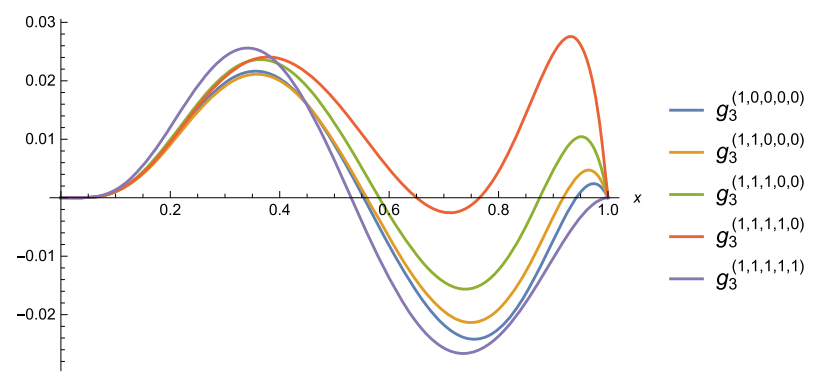

(a) $g_{3}$

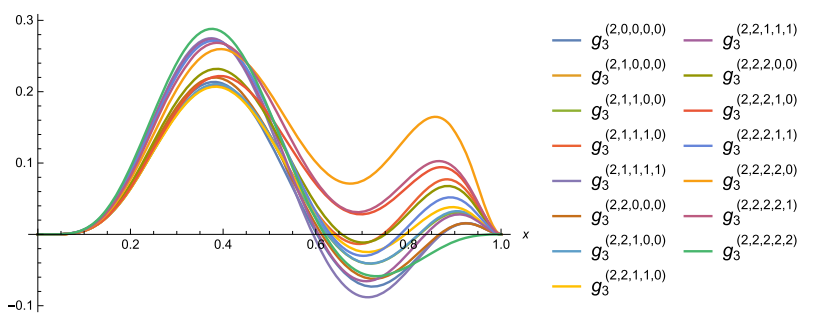

(b) $g_{3}$

FIG. 9. Full structure function $g_{3}$ as a function of the Bjorken parameter $x$ obtained from the twist- 4 spin- $1 / 2$ fermionic operators $\mathcal{O}_{k=1}^{I_{1},(6)}$ with $\left(1, l_{4}, l_{3}, l_{2}, l_{1}\right)_{a=1}$ (a) and in (b) from the twist-5 spin-1/2 fermionic operators $\mathcal{O}_{k=2}^{I_{2},(6)}$ with $\left(2, l_{4}, l_{3}, l_{2}, l_{1}\right)_{a=1}$.

\section{APPENDIX D: SPHERICAL HARMONICS}

In this Appendix some of the spinor spherical harmonics used to calculate the structure functions are explicitly written. In order to build them we have employed the formalism proposed in Ref. [76].

\section{The case with $\tau=4\left(l_{5}=1\right)$}

We first list the spinor spherical harmonics with $\mathcal{Q}=1 / 2$. Notice that $\Theta_{(1,0,0,0,0)_{a=1}}^{-}$is given in Sec. III A in Eq. (3.35):

$$
\begin{aligned}
& \Theta_{(1,1,0,0,0)_{a=1}}^{-}=\frac{\sqrt{3} e^{-i \mathcal{Q} \theta_{1}}}{\sqrt{10} \pi^{3 / 2}}\left[\begin{array}{c}
e^{-i \frac{1}{2}\left(\theta_{3}-\theta_{5}\right)} \cos \left(\frac{\theta_{2}}{2}\right) \cos \left(\frac{\theta_{4}}{2}\right) \sin \left(\theta_{5}\right)\left(1-5 \cos \left(\theta_{4}\right)\right) \\
e^{i \frac{1}{2}\left(\theta_{3}+\theta_{5}\right)} \sin \left(\frac{\theta_{2}}{2}\right) \cos \left(\frac{\theta_{4}}{2}\right) \sin \left(\theta_{5}\right)\left(-1+5 \cos \left(\theta_{4}\right)\right) \\
e^{-i \frac{1}{2}\left(\theta_{3}+\theta_{5}\right)} \cos \left(\frac{\theta_{2}}{2}\right) \sin \left(\frac{\theta_{4}}{2}\right) \sin \left(\theta_{5}\right)\left(1+5 \cos \left(\theta_{4}\right)\right) \\
e^{-i \frac{1}{2}\left(-\theta_{3}+\theta_{5}\right)} \sin \left(\frac{\theta_{2}}{2}\right) \sin \left(\frac{\theta_{4}}{2}\right) \sin \left(\theta_{5}\right)\left(1+5 \cos \left(\theta_{4}\right)\right)
\end{array}\right], \\
& \Theta_{(1,1,1,0,0)_{a=1}}^{-}=\frac{\sqrt{2} e^{-i \mathcal{Q} \theta_{1}}}{\pi^{3 / 2}}\left[\begin{array}{c}
e^{-i \frac{1}{2}\left(\theta_{3}-\theta_{5}\right)} \cos \left(\frac{\theta_{2}}{2}\right) \cos ^{2}\left(\frac{\theta_{4}}{2}\right) \sin \left(\frac{\theta_{4}}{2}\right) \sin \left(\theta_{5}\right)\left(-3 i \cos \left(\theta_{3}\right)-\sin \left(\theta_{3}\right)\right) \\
e^{i \frac{1}{2}\left(\theta_{3}+\theta_{5}\right)} \sin \left(\frac{\theta_{2}}{2}\right) \cos ^{2}\left(\frac{\theta_{4}}{2}\right) \sin \left(\frac{\theta_{4}}{2}\right) \sin \left(\theta_{5}\right)\left(3 i \cos \left(\theta_{3}\right)+\sin \left(\theta_{3}\right)\right) \\
e^{-i \frac{1}{2}\left(\theta_{3}+\theta_{5}\right)} \cos \left(\frac{\theta_{2}}{2}\right) \sin ^{2}\left(\frac{\theta_{4}}{2}\right) \cos \left(\frac{\theta_{4}}{2}\right) \sin \left(\theta_{5}\right)\left(-3 i \cos \left(\theta_{3}\right)-\sin \left(\theta_{3}\right)\right) \\
e^{-i \frac{1}{2}\left(-\theta_{3}+\theta_{5}\right)} \sin \left(\frac{\theta_{2}}{2}\right) \sin ^{2}\left(\frac{\theta_{4}}{2}\right) \cos \left(\frac{\theta_{4}}{2}\right) \sin \left(\theta_{5}\right)\left(3 i \cos \left(\theta_{3}\right)-\sin \left(\theta_{3}\right)\right)
\end{array}\right], \\
& \frac{\Theta_{(1,1,1,1,0)_{a=1}}^{-}}{\frac{e^{-i 2 \theta_{1}}}{\pi^{3 / 2}}}=\left[\begin{array}{c}
\sin \left(\theta_{2}\right)\left(1-3 \cos \left(\theta_{2}\right)\right) \csc \left(\frac{\theta_{2}}{2}\right) \sin \left(\frac{\theta_{3}}{2}\right) \sin \left(\theta_{3}\right)\left(\cot \left(\frac{\theta_{3}}{2}\right)-i\right) \sin ^{2}\left(\theta_{4}\right) \csc \left(\frac{\theta_{4}}{2}\right) \sin \left(\frac{\theta_{5}}{2}\right) \sin \left(\theta_{5}\right)\left(\cot \left(\frac{\theta_{5}}{2}\right)+i\right) \\
2 \sin \left(\frac{\theta_{2}}{2}\right)\left(3 \cos \left(\theta_{2}\right)+1\right) \sin \left(\frac{\theta_{3}}{2}\right) \sin \left(\theta_{3}\right)\left(\cot \left(\frac{\theta_{3}}{2}\right)+i\right) \sin ^{2}\left(\theta_{4}\right) \csc \left(\frac{\theta_{4}}{2}\right) \sin \left(\frac{\theta_{5}}{2}\right) \sin \left(\theta_{5}\right)\left(\cot \left(\frac{\theta_{5}}{2}\right)+i\right) \\
2 \sin \left(\theta_{2}\right)\left(3 \cos \left(\theta_{2}\right)-1\right) \csc \left(\frac{\theta_{2}}{2}\right) \sin \left(\frac{\theta_{3}}{2}\right) \sin \left(\theta_{3}\right)\left(\cot \left(\frac{\theta_{3}}{2}\right)-i\right) \sin \left(\frac{\theta_{4}}{2}\right) \sin \left(\theta_{4}\right) \sin \left(\frac{\theta_{5}}{2}\right) \sin \left(\theta_{5}\right)\left(\cot \left(\frac{\theta_{5}}{2}\right)-i\right) \\
-4 \sin \left(\frac{\theta_{2}}{2}\right)\left(3 \cos \left(\theta_{2}\right)+1\right) \sin \left(\frac{\theta_{3}}{2}\right) \sin \left(\theta_{3}\right)\left(\cot \left(\frac{\theta_{3}}{2}\right)+i\right) \sin \left(\frac{\theta_{4}}{2}\right) \sin \left(\theta_{4}\right) \sin \left(\frac{\theta_{5}}{2}\right) \sin \left(\theta_{5}\right)\left(\cot \left(\frac{\theta_{5}}{2}\right)-i\right)
\end{array}\right] .
\end{aligned}
$$


Next, for $\mathcal{Q}=3 / 2$ we have

$$
\Theta_{(1,1,1,1,1)_{a=1}}^{-}=\frac{\sqrt{3} e^{-i \mathcal{Q} \theta_{1}}}{4 \pi^{3 / 2}}\left[\begin{array}{c}
i e^{-\frac{1}{2} i\left(\theta_{3}-\theta_{5}\right)} \sin ^{2}\left(\theta_{2}\right) \csc \left(\frac{\theta_{2}}{2}\right) \sin \left(\theta_{3}\right) \sin ^{2}\left(\theta_{4}\right) \csc \left(\frac{\theta_{4}}{2}\right) \sin \left(\theta_{5}\right) \\
2 i e^{\frac{1}{2}\left(\theta_{3}+\theta_{5}\right)} \sin \left(\frac{\theta_{2}}{2}\right) \sin \left(\theta_{2}\right) \sin \left(\theta_{3}\right) \sin ^{2}\left(\theta_{4}\right) \csc \left(\frac{\theta_{4}}{2}\right) \sin \left(\theta_{5}\right) \\
2 i \sin ^{2}\left(\theta_{2}\right) \csc \left(\frac{\theta_{2}}{2}\right) \sin \left(\frac{\theta_{3}}{2}\right) \sin \left(\theta_{3}\right)\left(\cot \left(\frac{\theta_{3}}{2}\right)-i\right) \sin \left(\frac{\theta_{4}}{2}\right) \sin \left(\theta_{4}\right) \sin \left(\frac{\theta_{5}}{2}\right) \sin \left(\theta_{5}\right)\left(\cot \left(\frac{\theta_{5}}{2}\right)-i\right) \\
4 i e^{-\frac{1}{2}\left(-\theta_{3}+\theta_{5}\right)} \sin \left(\frac{\theta_{2}}{2}\right) \sin \left(\theta_{2}\right) \sin \left(\theta_{3}\right) \sin \left(\frac{\theta_{4}}{2}\right) \sin \left(\theta_{4}\right) \sin \left(\theta_{5}\right)
\end{array}\right] .
$$

\section{The case with $\tau=5\left(l_{5}=2\right)$}

There are ten spinor spherical harmonics with $\mathcal{Q}=1 / 2$, four with $\mathcal{Q}=3 / 2$ and only one with $\mathcal{Q}=5 / 2$. We only show a few examples for each charge.

For $\mathcal{Q}=1 / 2$ we display the spinor spherical harmonic $(2,0,0,0,0)_{a=1}$ as follows:

$$
\begin{aligned}
& \Theta_{(2,0,0,0,0)_{a=1}}^{-}=\frac{\sqrt{3} e^{-i \mathcal{Q} \theta_{1}}}{\sqrt{5} \pi^{3 / 2}}\left[\begin{array}{c}
i e^{-\frac{1}{2} i\left(\theta_{3}-\theta_{5}\right)} \cos \left(\frac{\theta_{2}}{2}\right) \cos \left(\frac{\theta_{4}}{2}\right)\left(i \sin \left(2 \theta_{5}\right)+3 \cos \left(2 \theta_{5}\right)+2\right) \\
e^{-\frac{1}{2} i\left(-\theta_{3}-\theta_{5}\right)} \sin \left(\frac{\theta_{2}}{2}\right) \cos \left(\frac{\theta_{4}}{2}\right)\left(\sin \left(2 \theta_{5}\right)-3 i \cos \left(2 \theta_{5}\right)-2 i\right) \\
-i e^{-\frac{1}{2} i\left(\theta_{3}+\theta_{5}\right)} \cos \left(\frac{\theta_{2}}{2}\right) \sin \left(\frac{\theta_{4}}{2}\right)\left(-i \sin \left(2 \theta_{5}\right)+3 \cos \left(2 \theta_{5}\right)+2\right) \\
e^{-\frac{1}{2} i\left(-\theta_{3}+\theta_{5}\right)} \sin \left(\frac{\theta_{2}}{2}\right) \sin \left(\frac{\theta_{4}}{2}\right)\left(\sin \left(2 \theta_{5}\right)+3 i \cos \left(2 \theta_{5}\right)+2 i\right)
\end{array}\right], \\
& \Theta_{(2,1,0,0,0)_{a=1}}^{-}=\frac{\sqrt{3} e^{-i \mathcal{Q} \theta_{1}}}{\sqrt{70} \pi^{3 / 2}}\left[\begin{array}{c}
i e^{-\frac{1}{2} i\left(\theta_{3}-\theta_{5}\right)} \cos \left(\frac{\theta_{2}}{2}\right) \cos \left(\frac{\theta_{4}}{2}\right)\left(5 \cos \left(\theta_{4}\right)-1\right) \sin \left(\theta_{5}\right)\left(7 \cos \left(\theta_{5}\right)+i \sin \left(\theta_{5}\right)\right) \\
e^{-\frac{1}{2} i\left(-\theta_{3}-\theta_{5}\right)} \sin \left(\frac{\theta_{2}}{2}\right) \cos \left(\frac{\theta_{4}}{2}\right)\left(5 \cos \left(\theta_{4}\right)-1\right) \sin \left(\theta_{5}\right)\left(\sin \left(\theta_{5}\right)-7 i \cos \left(\theta_{5}\right)\right) \\
-e^{-\frac{1}{2} i\left(\theta_{3}+\theta_{5}\right)} \cos \left(\frac{\theta_{2}}{2}\right) \sin \left(\frac{\theta_{4}}{2}\right)\left(5 \cos \left(\theta_{4}\right)+1\right) \sin \left(\theta_{5}\right)\left(\sin \left(\theta_{5}\right)+7 i \cos \left(\theta_{5}\right)\right) \\
e^{-\frac{1}{2} i\left(-\theta_{3}+\theta_{5}\right)} \sin \left(\frac{\theta_{2}}{2}\right) \sin \left(\frac{\theta_{4}}{2}\right)\left(5 \cos \left(\theta_{4}\right)+1\right) \sin \left(\theta_{5}\right)\left(\sin \left(\theta_{5}\right)+7 i \cos \left(\theta_{5}\right)\right)
\end{array}\right] .
\end{aligned}
$$

For charge $\mathcal{Q}=3 / 2$ we write the example:

$$
\frac{\Theta_{(2,2,1,1,1)_{1}}^{-}}{\frac{e^{-i Q \theta_{1}}}{\sqrt{7} \pi^{3 / 2}}}=\left[\begin{array}{c}
4 i e^{-\frac{1}{2} i\left(\theta_{3}-\theta_{5}\right)} \sin \left(\frac{\theta_{2}}{2}\right) \cos ^{2}\left(\frac{\theta_{2}}{2}\right) \sin \left(\theta_{3}\right)\left(7 \sin \left(\frac{3 \theta_{4}}{2}\right)-9 \sin \left(\frac{\theta_{4}}{2}\right)\right) \cos ^{2}\left(\frac{\theta_{4}}{2}\right) \sin ^{2}\left(\theta_{5}\right) \\
\sin \left(\frac{\theta_{2}}{2}\right) \sin \left(\theta_{2}\right) \sin \left(\frac{\theta_{3}}{2}\right) \sin \left(\theta_{3}\right)\left(\cot \left(\frac{\theta_{3}}{2}\right)+i\right) \sin ^{2}\left(\theta_{4}\right)\left(7 \cos \left(\theta_{4}\right)-1\right) \csc \left(\frac{\theta_{4}}{2}\right) \sin \left(\frac{\theta_{5}}{2}\right) \sin ^{2}\left(\theta_{5}\right)\left(1-i \cot \left(\frac{\theta_{5}}{2}\right)\right) \\
\sin ^{2}\left(\theta_{2}\right) \csc \left(\frac{\theta_{2}}{2}\right) \sin \left(\frac{\theta_{3}}{2}\right) \sin \left(\theta_{3}\right)\left(-1-i \cot \left(\frac{\theta_{3}}{2}\right)\right) \sin \left(\frac{\theta_{4}}{2}\right) \sin \left(\theta_{4}\right)\left(7 \cos \left(\theta_{4}\right)+1\right) \sin \left(\frac{\theta_{5}}{2}\right) \sin ^{2}\left(\theta_{5}\right)\left(\cot \left(\frac{\theta_{5}}{2}\right)-i\right) \\
2 \sin \left(\frac{\theta_{2}}{2}\right) \sin \left(\theta_{2}\right) \sin \left(\frac{\theta_{3}}{2}\right) \sin \left(\theta_{3}\right)\left(\cot \left(\frac{\theta_{3}}{2}\right)+i\right) \sin \left(\frac{\theta_{4}}{2}\right) \sin \left(\theta_{4}\right)\left(7 \cos \left(\theta_{4}\right)+1\right) \sin \left(\frac{\theta_{5}}{2}\right) \sin ^{2}\left(\theta_{5}\right)\left(1+i \cot \left(\frac{\theta_{5}}{2}\right)\right)
\end{array}\right] .
$$


Finally, for $\mathcal{Q}=5 / 2$ we have

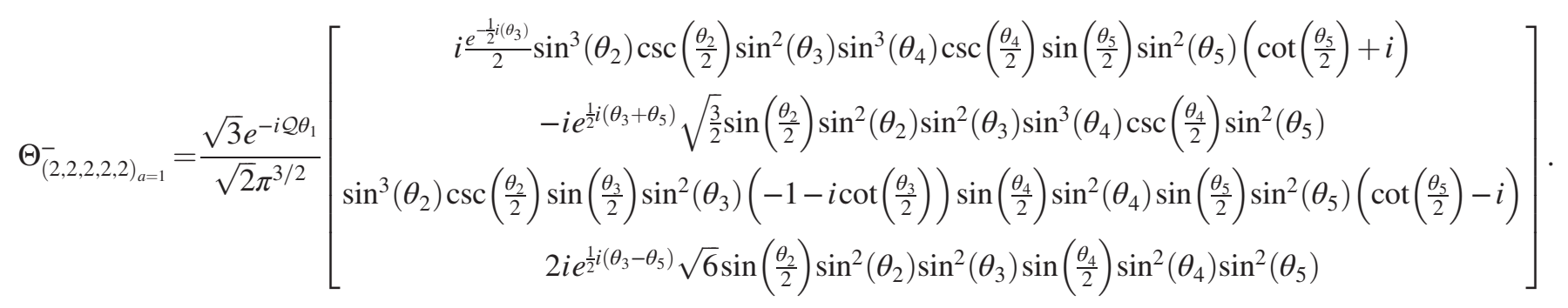

[1] J. M. Maldacena, The large N limit of superconformal field theories and supergravity, Int. J. Theor. Phys. 38, 1113 (1999).

[2] S. S. Gubser, I. R. Klebanov, and A. M. Polyakov, Gauge theory correlators from noncritical string theory, Phys. Lett. B 428, 105 (1998).

[3] E. Witten, Anti-de Sitter space and holography, Adv. Theor. Math. Phys. 2, 253 (1998).

[4] J. Polchinski and M. J. Strassler, Deep inelastic scattering and gauge/string duality, J. High Energy Phys. 05 (2003) 012.

[5] J. Gao and B. Xiao, Polarized deep inelastic and elastic scattering from gauge/string duality, Phys. Rev. D 80, 015025 (2009).

[6] J. H. Gao and Z. G. Mou, Polarized deep inelastic scattering off the neutron from gauge/string duality, Phys. Rev. D 81, 096006 (2010).

[7] C. A. Ballon Bayona, H. Boschi-Filho, and N. R. F. Braga, Deep inelastic scattering from gauge string duality in the soft wall model, J. High Energy Phys. 03 (2008) 064.

[8] C. A. Ballon Bayona, H. Boschi-Filho, and N. R. F. Braga, Deep inelastic scattering in holographic AdS/QCD models, Nucl. Phys. B, Proc. Suppl. 199, 97 (2010).

[9] N. Kovensky, G. Michalski, and M. Schvellinger, Deep inelastic scattering from polarized spin- $1 / 2$ hadrons at low $x$ from string theory, J. High Energy Phys. 10 (2018) 084.

[10] D. Jorrin, G. Michalski, and M. Schvellinger, Spin-1/2 fermionic operators of $\mathcal{N}=4$ SYM theory and DIS from type IIB supergravity, J. High Energy Phys. 06 (2020) 063.

[11] M. Kruczenski, D. Mateos, R. C. Myers, and D. J. Winters, Meson spectroscopy in AdS/CFT with flavor, J. High Energy Phys. 07 (2003) 049.

[12] T. Sakai and S. Sugimoto, Low energy hadron physics in holographic QCD, Prog. Theor. Phys. 113, 843 (2005).

[13] M. Kruczenski, D. Mateos, R. C. Myers, and D. J. Winters, Towards a holographic dual of large N(c) QCD, J. High Energy Phys. 05 (2004) 041.

[14] C. A. Ballon Bayona, H. Boschi-Filho, and N. R. F. Braga, Deep inelastic scattering from gauge string duality in D3-D7 brane model, J. High Energy Phys. 09 (2008) 114.
[15] C. A. Ballon Bayona, H. Boschi-Filho, N. R. F. Braga, and M. A. C. Torres, Deep inelastic scattering for vector mesons in holographic D4-D8 model, J. High Energy Phys. 10 (2010) 055.

[16] C. A. B. Bayona, H. Boschi-Filho, N. R. F. Braga, M. Ihl, and M. A.C. Torres, Generalized baryon form factors and proton structure functions in the Sakai-Sugimoto model, Nucl. Phys. B866, 124 (2013).

[17] E. Koile, S. Macaluso, and M. Schvellinger, Deep inelastic scattering from holographic spin-one hadrons, J. High Energy Phys. 02 (2012) 103.

[18] E. Koile, S. Macaluso, and M. Schvellinger, Deep inelastic scattering structure functions of holographic spin-1 hadrons with $N_{f} \geq 1$, J. High Energy Phys. 01 (2014) 166.

[19] E. Koile, N. Kovensky, and M. Schvellinger, Hadron structure functions at small $x$ from string theory, J. High Energy Phys. 05 (2015) 001.

[20] E. Koile, N. Kovensky, and M. Schvellinger, Deep inelastic scattering cross sections from the gauge/string duality, J. High Energy Phys. 12 (2015) 009.

[21] D. Jorrin, N. Kovensky, and M. Schvellinger, Towards 1/N corrections to deep inelastic scattering from the gauge/ gravity duality, J. High Energy Phys. 04 (2016) 113.

[22] D. Jorrin, M. Schvellinger, and N. Kovensky, Deep inelastic scattering off scalar mesons in the $1 / \mathrm{N}$ expansion from the D3D7-brane system, J. High Energy Phys. 12 (2016) 003.

[23] N. Kovensky, G. Michalski, and M. Schvellinger, $1 / N$ corrections to $F_{1}$ and $F_{2}$ structure functions of vector mesons from holography, Phys. Rev. D 99, 046005 (2019).

[24] R. C. Brower, M. J. Strassler, and C. I. Tan, On the Pomeron at large 't Hooft coupling, J. High Energy Phys. 03 (2009) 092.

[25] R. C. Brower, M. J. Strassler, and C. I. Tan, On the eikonal approximation in AdS space, J. High Energy Phys. 03 (2009) 050.

[26] L. Cornalba, M. S. Costa, J. Penedones, and R. Schiappa, Eikonal approximation in AdS/CFT: Conformal partial waves and finite $\mathrm{N}$ four-point functions, Nucl. Phys. B767, 327 (2007). 
[27] L. Cornalba, M. S. Costa, and J. Penedones, Eikonal approximation in AdS/CFT: Resumming the gravitational loop expansion, J. High Energy Phys. 09 (2007) 037.

[28] M.S. Costa and M. Djuric, Deeply virtual Compton scattering from gauge/gravity duality, Phys. Rev. D 86, 016009 (2012).

[29] A. Watanabe and K. Suzuki, Transition from soft- to hardPomeron in the structure functions of hadrons at small- $x$ from holography, Phys. Rev. D 86, 035011 (2012).

[30] M.S. Costa, M. Djuric, and N. Evans, Vector meson production at low $\mathrm{x}$ from gauge/gravity duality, J. High Energy Phys. 09 (2013) 084.

[31] N. Kovensky, G. Michalski, and M. Schvellinger, DIS off glueballs from string theory: The role of the chiral anomaly and the Chern-Simons term, J. High Energy Phys. 04 (2018) 118.

[32] Y. Hatta, E. Iancu, and A. H. Mueller, Deep inelastic scattering off a $N=4$ SYM plasma at strong coupling, J. High Energy Phys. 01 (2008) 063.

[33] B. Hassanain and M. Schvellinger, Holographic current correlators at finite coupling and scattering off a supersymmetric plasma, J. High Energy Phys. 04 (2010) 012.

[34] M.F. Paulos, Higher derivative terms including the Ramond-Ramond five-form, J. High Energy Phys. 10 (2008) 047.

[35] M. Anselmino, A. Efremov, and E. Leader, The theory and phenomenology of polarized deep inelastic scattering, Phys. Rep. 261, 1 (1995); , Erratum, Phys. Rep. 281, 399 (1997).

[36] B. Lampe and E. Reya, Spin physics and polarized structure functions, Phys. Rep. 332, 1 (2000).

[37] O. Aharony, S. S. Gubser, J. M. Maldacena, H. Ooguri, and Y. Oz, Large $\mathrm{N}$ field theories, string theory and gravity, Phys. Rep. 323, 183 (2000).

[38] E. D'Hoker and D. Z. Freedman, Supersymmetric gauge theories and the AdS/CFT correspondence, arXiv:hep-th/ 0201253.

[39] H. J. Kim, L. J. Romans, and P. van Nieuwenhuizen, The mass spectrum of chiral $N=2 D=10$ supergravity on S**5, Phys. Rev. D 32, 389 (1985).

[40] P. van Nieuwenhuizen, The compactification of IIB supergravity on $S_{5}$ revisited, arXiv:1206.2667.

[41] P. van Nieuwenhuizen, The Kaluza-Klein program and supergravity the compactification of type 2B supergravity revisited, in The New Physics Frontiers in the LHC-2 Era, Lectures at the International School for Subnuclear Physics (2019) 247-279, https://doi.org/10.1142/ 9789811206856_0009.

[42] G. Dall'Agata, K. Lechner, and M. Tonin, $D=10, N=$ IIB supergravity: Lorentz invariant actions and duality, J. High Energy Phys. 07 (1998) 017.

[43] J. H. Schwarz and P. C. West, Symmetries and transformations of chiral $N=2 D=10$ supergravity, Phys. Lett. 126B, 301 (1983).

[44] P. S. Howe and P. C. West, The complete $N=2, D=10$ supergravity, Nucl. Phys. B238, 181 (1984).

[45] J.H. Schwarz, Covariant field equations of chiral $N=2$ $D=10$ supergravity, Nucl. Phys. B226, 269 (1983).

[46] W. Mueck and K. S. Viswanathan, Conformal field theory correlators from classical field theory on anti-de Sitter space. 2. Vector and spinor fields, Phys. Rev. D 58, 106006 (1998).

[47] K. A. Mamo and I. Zahed, Diffractive photoproduction of $J / \psi$ and $\Upsilon$ using holographic QCD: Gravitational form factors and GPD of gluons in the proton, Phys. Rev. D 101, 086003 (2020).

[48] A. V. Manohar, An introduction to spin dependent deep inelastic scattering, arXiv:hep-ph/9204208.

[49] A. V. Kotikov and L. N. Lipatov, DGLAP and BFKL equations in the $N=4$ supersymmetric gauge theory, Nucl. Phys. B661, 19 (2003).

[50] L. Bianchi, V. Forini, and A. V. Kotikov, On DIS Wilson coefficients in $N=4$ super Yang-Mills theory, Phys. Lett. B 725, 394 (2013).

[51] T. Gutsche, V. E. Lyubovitskij, I. Schmidt, and A. Vega, Light-front quark model consistent with Drell-Yan-West duality and quark counting rules, Phys. Rev. D 89, 054033 (2014); , Erratum, Phys. Rev. D 92, 019902 (2015).

[52] S. D. Drell and T. M. Yan, Connection of Elastic Electromagnetic Nucleon Form-Factors at Large Q**2 and Deep Inelastic Structure Functions Near Threshold, Phys. Rev. Lett. 24, 181 (1970).

[53] G. B. West, Phenomenological Model for the Electromagnetic Structure of the Proton, Phys. Rev. Lett. 24, 1206 (1970).

[54] S. J. Brodsky and G. R. Farrar, Scaling Laws at Large Transverse Momentum, Phys. Rev. Lett. 31, 1153 (1973).

[55] V. A. Matveev, R. M. Muradian, and A. N. Tavkhelidze, Automodellism in the large-angle elastic scattering and structure of hadrons, Lett. Nuovo Cimento 7, 719 (1973).

[56] T. Gutsche, V. E. Lyubovitskij, I. Schmidt, and A. Vega, Nucleon structure in a light-front quark model consistent with quark counting rules and data, Phys. Rev. D 91, 054028 (2015).

[57] G. F. de Teramond, T. Liu, R. Sabbir Sufian, H. G. Dosch, S. J. Brodsky, and A. Deur (HLFHS Collaboration), Universality of Generalized Parton Distributions in LightFront Holographic QCD, Phys. Rev. Lett. 120, 182001 (2018).

[58] R. C. Brower, J. Polchinski, M. J. Strassler, and C. I. Tan, The Pomeron and gauge/string duality, J. High Energy Phys. 12 (2007) 005.

[59] L. W. Whitlow, E. M. Riordan, S. Dasu, S. Rock, and A. Bodek, Precise measurements of the proton and deuteron structure functions from a global analysis of the SLAC deep inelastic electron scattering cross sections, Phys. Lett. B 282, 475 (1992).

[60] A. C. Benvenuti et al. (BCDMS Collaboration), A high statistics measurement of the proton structure functions $\mathrm{F}(2)\left(\mathrm{x}, \mathrm{Q}^{* * 2}\right)$ and $\mathrm{R}$ from deep inelastic muon scattering at high Q**2, Phys. Lett. B 223, 485 (1989).

[61] E. Folco Capossoli, M. A. Martín Contreras, D. Li, A. Vega, and $\mathrm{H}$. Boschi-Filho, Proton structure functions from an AdS/QCD model with a deformed background, Phys. Rev. D 102, 086004 (2020).

[62] G. F. de Teramond and S. J. Brodsky, Hadronic Spectrum of a Holographic Dual of QCD, Phys. Rev. Lett. 94, 201601 (2005). 
[63] T. Gutsche, V. E. Lyubovitskij, I. Schmidt, and A. Vega, Nucleon structure including high Fock states in AdS/QCD, Phys. Rev. D 86, 036007 (2012).

[64] I. R. Klebanov and E. Witten, Superconformal field theory on three-branes at a Calabi-Yau singularity, Nucl. Phys. B536, 199 (1998).

[65] A. Ceresole, G. Dall'Agata, R. D'Auria, and S. Ferrara, Spectrum of type IIB supergravity on $\mathrm{AdS}_{5} \times T^{1,1}$ : Predictions on $N=1$ SCFT's, Phys. Rev. D 61, 066001 (2000).

[66] A. Ceresole, G. Dall'Agata, R. D'Auria, and S. Ferrara, Superconformal field theories from IIB spectroscopy on $\mathrm{AdS}_{5} \times T^{1,1}$, Classical Quantum Gravity 17, 1017 (2000).

[67] N. Seiberg, Five-dimensional SUSY field theories, nontrivial fixed points and string dynamics, Phys. Lett. B 388, 753 (1996).

[68] A. Brandhuber and Y. Oz, The D-4-D-8 brane system and five-dimensional fixed points, Phys. Lett. B 460, 307 (1999).

[69] L. J. Romans, Massive $\mathrm{N}=2$ a supergravity in ten dimensions, Phys. Lett. 169B, 374 (1986).
[70] R. D'Auria, S. Ferrara, and S. Vaula, Matter coupled $\mathrm{F}(4)$ supergravity and the $\operatorname{AdS}(6) / \mathrm{CFT}(5)$ correspondence, J. High Energy Phys. 10 (2000) 013.

[71] C. Nunez, I. Y. Park, M. Schvellinger, and T. A. Tran, Supergravity duals of gauge theories from F(4) gauged supergravity in six dimensions, J. High Energy Phys. 04 (2001) 025.

[72] S. Ferrara and E. Sokatchev, Conformal superfields and BPS states in $\operatorname{AdS}(4 / 7)$ geometries, Int. J. Mod. Phys. B 14, 2315 (2000).

[73] S. Ferrara and E. Sokatchev, Representations of superconformal algebras in the $\operatorname{AdS}(7 / 4) / C F T(6 / 3)$ correspondence, J. Math. Phys. (N.Y.) 42, 3015 (2001).

[74] E. Sezgin, 11D Supergravity on $\mathrm{AdS}_{4} \times S^{7}$ versus $\mathrm{AdS}_{7} \times S^{4}$, J. Phys. A 53, 364003 (2020).

[75] U. Gursoy, C. Nunez, and M. Schvellinger, RG flows from spin(7), CY 4 fold and HK manifolds to AdS, Penrose limits and pp waves, J. High Energy Phys. 06 (2002) 015.

[76] R. Camporesi and A. Higuchi, On the eigenfunctions of the Dirac operator on spheres and real hyperbolic spaces, J. Geom. Phys. 20, 1 (1996). 